\title{
Nucleosynthesis-relevant conditions in neutrino-driven supernova outflows
}

\section{Spherically symmetric hydrodynamic simulations}

\author{
A. Arcones, H.-Th. Janka, and L. Scheck
}

\begin{abstract}
Max-Planck-Institut für Astrophysik, Karl-Schwarzschild-Straße 1, 85741 Garching, Germany e-mail: thj@mpa-garching.mpg.de
\end{abstract}

Received 20 December 2006 / Accepted 15 March 2007

ABSTRACT

\begin{abstract}
We investigate the behavior and consequences of the reverse shock that terminates the supersonic expansion of the baryonic wind which is driven by neutrino heating off the surface of (non-magnetized) new-born neutron stars in supernova cores. To this end we perform long-time hydrodynamic simulations in spherical symmetry. In agreement with previous relativistic wind studies, we find that the neutrino-driven outflow accelerates to supersonic velocities and in case of a compact, $\sim 1.4 M_{\odot}$ (gravitational mass) neutron star with a radius of about $10 \mathrm{~km}$, the wind reaches entropies of about $100 k_{\mathrm{B}}$ per nucleon. The wind, however, is strongly influenced by the environment of the supernova core. It is decelerated and shock-heated abruptly by a termination shock that forms when the supersonic outflow collides with the slower preceding supernova ejecta. The radial position of this reverse shock varies with time and depends on the strength of the neutrino wind and the explosion conditions in progenitor stars with different masses and structure. Its basic properties and behavior can be understood by simple analytic considerations. We demonstrate that the entropy of the matter going through the reverse shock can increase to a multiple of the asymptotic wind value. Seconds after the onset of the explosion it therefore can exceed $400 k_{\mathrm{B}}$ per nucleon in low-mass progenitors around $10 M_{\odot}$, where the supernova shock and the reverse shock propagate outward quickly. The temperature of the shocked wind has typically dropped to about or less than $10^{9} \mathrm{~K}$, and density and temperature in the shock-decelerated matter continue to decrease only very slowly. For more massive progenitors with bigger and denser metal cores, the explosion expands more slowly so that the termination shock stays at smaller radii and affects the wind at higher temperatures and densities. In this case the termination shock might play a non-negligible, strongly time- and progenitordependent role in discussing supernova nucleosynthesis.
\end{abstract}

Key words. supernovae: general - neutrinos - nuclear reactions, nucleosynthesis, abundances - hydrodynamics

Neutron stars are born as extremely hot and dense remnants at the center of exploding massive stars. Shortly after their formation they heat up to temperatures that can reach tens of $\mathrm{MeV}$ and become even higher than $50 \mathrm{MeV}$ for soft nuclear equations of state (e.g., Burrows \& Lattimer 1986; Keil \& Janka 1995; Pons et al. 1999). Their gravitational binding energy is carried away by neutrinos, which are abundantly produced at such conditions. On a timescale of seconds these neutrinos diffuse out of the interior of the star and escape from their mean surface of last scattering, the neutrinosphere. By the associated energy and lepton number loss, the hot, still proton-rich and neutrino-opaque proto-neutron star thus evolves to the final cold, neutron-rich and neutrino-transparent remnant during roughly the first minute of its life.

Outside of the neutrinosphere the radiated neutrinos, which have typical energies of 10-20 MeV, travel through a layer with a very steep density gradient and decreasing temperature. It is unavoidable that the residual interactions of the high-energy neutrinos with the cooler stellar matter deposit energy in this region. This energy transfer does not allow the "surface" layers of the hot, neutrino-cooling neutron star to remain in hydrostatic equilibrium, but leads to mass loss at a low rate in a neutrinodriven outflow of baryonic matter (Duncan et al. 1986; Woosley $\&$ Baron 1992). This outflow, the so-called "neutrino-driven wind", unavoidably accompanies the birth of a hot, neutrinocooling neutron star, independent of the details of the not finally understood supernova explosion mechanism (in case of sufficiently strong magnetic fields, however, there my be magnetically driven outflow instead of a thermally-driven wind; see Metzger et al. 2006). The mass loss of the nascent neutron star begins after the supernova explosion has been launched and continues until the neutron star is essentially transparent to neutrinos. This flow of baryonic matter is a rapidly expanding and cooling high-entropy environment, in which an $\alpha$-rich freezeout can lead to the production of elements heavier than the iron group. For sufficiently large neutron excess, the $\alpha$-rich freezeout can merge smoothly into an r-process (Woosley \& Hoffman 1992; Meyer et al. 1992).

In fact, a number of parameters has been recognized to determine the possibility of r-process nucleosynthesis in the neutrinowind environment: The neutron-to-proton ratio in the wind, expressed in terms of the electron-to-baryon ratio or electron faction $Y_{\mathrm{e}}$; the expansion timescale, $\tau$, which decides how fast the temperature and density of the outflowing matter drop; and the wind entropy per nucleon, $s$, as a measure of the photonto-baryon ratio of the environment (Witti et al. 1994; Qian \& Woosley 1996; Hoffman et al. 1996). The entropy of the wind is typically tens to more than $100 k_{\mathrm{B}}$ per nucleon, making the wind environment a candidate for the so-called high-entropy r-process (Meyer et al. 1992; Meyer 1994). In addition, the mass loss rate decides whether the wind could be the major source of the observed galactic abundances of r-process material. These wind 
parameters depend on the neutron star properties, in particular on the gravitational field of the neutron star and thus its mass and radius, and on the neutrino emission of the neutron star, i.e., the time-dependent luminosities and spectra of the radiated neutrinos (Qian \& Woosley 1996). Since it is mainly the absorption of electron neutrinos, $v_{\mathrm{e}}$, and antineutrinos, $\bar{v}_{\mathrm{e}}$, on free neutrons and protons, respectively, which heats the stellar gas and is responsible for driving the mass loss and for setting the electron fraction in the ejected gas, the emission properties of these neutrinos are most relevant (but see Wanajo 2006 for a suggestion that additional energy input to the wind by $v \bar{v}$-annihilation in case of largely anisotropic neutrino emission from the nascent neutron star could lead to a decisive increase of the wind entropy).

Only in case the neutrino-driven outflow becomes supersonic beyond a critical point, the sonic point, it truly deserves the name "wind". In such wind solutions the physical conditions at the neutrinosphere and behind the supernova shock are causally disconnected. The presence of the sonic point unambiguously determines the solution for a given value of the driving luminosity. Wind solutions possess the highest ("critical") mass loss rate (and the lowest specific total energy of the ejected matter) for a given neutrino luminosity. Physical solutions with larger mass loss rates (and lower specific total energy) do not exist. Lower mass loss rates (higher specific total energies) correspond to "breeze solutions" (Takahashi \& Janka 1997; Otsuki et al. 2000). In these, the outflow velocity reaches a maximum and then decreases again to asymptote to zero at infinity. The whole region between the proto-neutron star surface and the outer boundary of the considered outflow is therefore in sonic contact. While wind solutions are characterized by a continuously rising velocity and decreasing temperature, the temperature of breezes level off to a constant value at large radii where the flow is dominated by internal instead of kinetic energy. This limiting value of the temperature at large distances from the neutron star is an additional characteristic parameter of breeze solutions.

Transsonic neutrino-driven winds in the context of supernova explosions and nucleosynthesis were investigated by means of hydrodynamic simulations (Woosley \& Baron 1992), analytic discussion (Qian \& Woosley 1996; Cardall \& Fuller 1997), and numerical solutions of the steady-state wind equations (Otsuki et al. 2000; Thompson et al. 2001). Otsuki et al. (2000) discussed the difference between winds and breezes, but like Wanajo et al. (2001) they concentrated on the subsonic solutions for their nucleosynthesis calculations, mainly because these allowed them to set a boundary value of the temperature at some large radius. This was understood to mimic the transition of the wind into a dense shell of ejecta behind the outgoing supernova shock, the presence of which hampered the free expansion of the wind. Such a behavior was found in calculations of supernova explosions by the Livermore group, which were employed in the r-process studies of Woosley \& Hoffman (1992), Woosley et al. (1994), and Hoffman et al. (1996), and also in hydrodynamic simulations of neutrino-driven outflows by Witti et al. (1994) and Takahashi et al. (1994), which were started from postbounce models provided by the Livermore group. The outflow trajectories in these simulations showed temperature and density declining asymptotically to nearly constant values, which were reached when the flow was gradually decelerated upon catching up with the slower, earlier ejecta behind the supernova shock. Sumiyoshi et al. (2000) and Terasawa et al. (2002) also referred to this behavior for using an artificially imposed constant pressure at the outer boundary in their Lagrangian hydrodynamic simulations of neutrino-driven mass ejection. The external pressure produced outflow deceleration similar to that found in the previous supernova models.

Applying modern, high-resolution shock-capturing schemes and a better numerical resolution to long-time hydrodynamic simulations of supernova explosions, Janka \& Müller (1995) and Burrows et al. (1995) (see also the more recent models of Buras et al. 2006b used for nucleosynthesis studies by Pruet et al. 2005) discovered the formation of a wind termination shock caused by the collision of a transsonic neutrino-driven wind with the dense, slower ejecta shell behind the supernova shock. So far, however, this reverse shock, which leads to an abrupt deceleration and shock heating of the fast wind, has not received much attention. Subtle, potentially significant effects in the r-process nucleosynthesis that may depend in interesting ways on the location of and strength of the reverse shock were found by Thompson et al. (2001). Although these authors mentioned a rather modest reheating of the wind material by the reverse shock passage (that causes a increase of the specific entropy of $10 k_{\mathrm{B}}$ per nucleon), they obtained a considerably enhanced production of third-peak r-process nuclei due to a slower postshock expansion and a significantly higher temperature $(0.05 \mathrm{MeV}$ instead of $0.01 \mathrm{MeV}$ for unshocked winds) at the time the r-process freeze-out happens. Also Wanajo et al. (2002), alluding to the possibility of a wind termination shock, introduced a freeze-out value $T_{\mathrm{f}}$ as the final temperature of the wind, i.e., they limited the temperature (and density) decrease in the supersonic wind by a chosen lower value. The choice of this temperature was, naturally, to some degree ad hoc, although Wanajo et al. (2002) justified it by nucleosynthesis considerations. A systematic and detailed exploration of the formation of the wind termination shock, of its hydrodynamical effects on the wind properties, and of its nucleosynthetic consequences, however, is still lacking.

In this paper we will study the time-dependent evolution of the wind termination shock in different progenitors with spherically symmetric (1D) models. For this purpose we perform simulations of neutrino-driven explosions, employing the approximations to the full supernova physics used in previous works (Scheck et al. 2004, 2006). The neutron star in our simulations is replaced by a contracting inner boundary at which neutrino luminosities are imposed such that supernova explosions with a typical explosion energy of $1-2 \times 10^{51} \mathrm{erg}=1-2$ bethe $(\mathrm{B})$ are triggered by neutrino heating. The subsequent explosion and evolution of the relic neutron star is followed until $10 \mathrm{~s}$ after core bounce. Varying the neutron star contraction, which depends on the incompletely known high-density equation of state, and the time-dependent neutrino emission from the forming neutron star, we will also investigate the sensitivity of the reverse shock effects on the neutrino-wind properties. Our results suggest that wind termination shocks are a robust, long-lasting feature in the supernova core just like the outgoing supernova shock and the neutrino-driven wind are. Of course, since a final understanding of the explosion mechanism of core-collapse supernovae is still missing (see, e.g. Buras et al. 2003, 2006a,b, and references therein) and because we excise the neutron star at the grid center instead of simulating its neutrino-cooling evolution and, moreover, make severe approximations to the important neutrino transport (see Sect. 2.2 and Appendix D in Scheck et al. 2006), our calculations will not be able to yield final answers. Nevertheless, our results are suitable for discussing fundamental properties of the wind termination shock and for developing a basic understanding of the role of this so far not well studied aspect of supernova explosions.

Our paper is structured in the following way. In Sect. 1 we will briefly describe the numerical approach taken in this work. 
In Sect. 2 we will present some comparisons we made with previous work, in particular testing our approximative treatment of relativistic effects. Section 3 contains our results for spherically symmetric simulations, providing a reference case and then investigating varied conditions (neutron star radius, neutrino luminosities) at the lower grid boundary and different progenitor stars. In addition, we will present an analytic discussion which allows one to basically understand the behavior and the properties of the wind termination shock. Section 5 will finish with a summary and conclusions.

\section{Numerical aspects}

\subsection{Hydrodynamics and neutrino treatment}

The simulations of this paper were carried out with the neutrinohydrodynamics code and the microphysics described by Scheck et al. (2006). The hydrodynamics module is a version of the Prometheus code which is based on a direct Eulerian implementation of the Piecewise Parabolic Method (PPM) of Colella \& Woodward (1984). It is a high-resolution shock capturing scheme and performs a conservative, explicit integration of the Newtonian hydrodynamics equations with third-order accuracy in space and second-order accuracy in time (see, e.g., Kifonidis et al. 2003 and references therein). General relativistic (GR) gravity is approximated by using an "effective relativistic potential" (for details, see Sect. 1.3). This approach accounts for the deeper gravitational well in GR, but still works in Minkowski spacetime and ignores the effects of relativistic kinematics. This was shown to yield excellent agreement with full GR calculations during the pre-explosion phase after core bounce (Liebendörfer et al. 2005; Marek et al. 2006) and will be demonstrated to also be a good approximation to full GR solutions of neutrino-driven winds in Sect. 2.

The equation of state used in the simulations presented here is valid below densities of roughly $10^{13} \mathrm{~g} \mathrm{~cm}^{-3}$ where non-ideal effects due to strong interactions between nucleons can be safely ignored. It was used before in the calculations by Janka \& Müller (1996), Kifonidis et al. (2003), and Scheck et al. (2006). Neutrons, protons, $\alpha$-particles and a representative heavy nucleus of the iron group (chosen to be ${ }^{54} \mathrm{Mn}$ ) are assumed to be nonrelativistic Boltzmann-gases in nuclear statistical equilibrium. Electrons and positrons are treated as Fermi-gases of arbitrary degeneracy and arbitrary degree of relativity, and photon contributions are included as well. Pressure and energy are corrected for Coulomb effects due to the electromagnetic interactions between nucleons and the surrounding sea of charged leptons.

The transport of neutrinos and antineutrinos of all flavors is based on a computationally very efficient, analytic integration along characteristics of the frequency-integrated zeroth-order moment equations of the Boltzmann equation for neutrino number and energy (for details, see the Appendix of Scheck et al. 2006). The integration yields the neutrino number and energy fluxes as functions of time and radius. Our approach thus accounts for the luminosity contributions due to the accretion on the forming neutron star. The neutrino spectra are assumed to have Fermi-Dirac shape with a spectral temperature that is determined from the ratio of neutrino-energy to neutrino-number flux. Therefore in general the spectral temperature is different from the local gas temperature. The closure of the neutrino number and energy equations is achieved by employing the flux factor $f(r, t)=F /(E c)$, which couples the local energy (or number) flux with the neutrino energy (or number) density. For $f(r, t)$ we use a prescribed function which was determined by fits to Monte Carlo transport results (Janka 1991). This yields a reasonably good approximation in the transparent and semi-transparent regimes but is not designed to accurately reproduce the diffusion limit at very high optical depths (where due to numerical reasons the applicability of the approach is anyway strongly constrained by the need of very fine grid zoning). The neutrino source terms in the transport equations and therefore the source terms for lepton number, energy, and momentum in the hydrodynamics equations include the most relevant neutrino-matter interactions (cf. Scheck et al. 2006).

We note that the steepening density gradient near the neutron star surface requires extremely fine grid zoning for getting converged results of the neutrino-driven outflow. We typically use about 1000 non-equidistant radial mesh points.

\subsection{Boundary treatment}

In our simulations we replace the inner core of the neutron star (usually roughly $1 M_{\odot}$ of baryonic matter) by an inner Lagrangian boundary of our grid, whose prescribed contraction is supposed to mimic the shrinking of the nascent neutron star as it loses energy and lepton number by neutrino emission. Using this inner boundary, which typically is located at a $v_{\mathrm{e}}$ optical depth of more than 100 and a density of $\rho_{\text {ib }} \gtrsim 10^{13} \mathrm{~g} \mathrm{~cm}^{-3}$, does not only allow us to apply the simple neutrino transport approximation described above, but also gives us the freedom to vary the time-evolution of the neutron star radius and of the core neutrino fluxes imposed at the inner grid boundary. This makes sense because both the equation of state of hot neutron star matter and the neutrino transport in nascent neutron stars are not finally understood. Changing the inner boundary conditions thus allows us to investigate the differences resulting from different explosion energies and timescales and from a different evolution of the neutrino-wind power in a given progenitor.

Three parameters serve us to describe the motion of the inner boundary: $R_{\mathrm{i}}, R_{\mathrm{f}}$, and $t_{0}$. The initial radius $R_{\mathrm{i}}$ is the radius of the inner core that we chose to excise from the postbounce models we start our simulations from, $R_{\mathrm{f}}$ is the final radius of this core for time $t \rightarrow \infty$, and $t_{0}$ is the timescale of an exponential contraction according to the expression

$R_{\mathrm{ib}}(t)=R_{\mathrm{f}}+\left(R_{\mathrm{i}}-R_{\mathrm{f}}\right) \mathrm{e}^{-t / t_{0}}$.

Our standard choice of $t_{0}=0.1 \mathrm{~s}$ reproduces the contraction of the excised core during the first few hundred milliseconds after bounce as found in full-scale supernova simulations with the equation of state of Lattimer \& Swesty (1991), using the energy-dependent neutrino transport of the VERTEX code (cf. Buras et al. 2006a and also Fig. 1 in Scheck et al. 2006).

In the simulations presented here we also explore the consequences of a different time-dependence of the neutrino luminosities imposed at the inner grid boundary (see Sects. 3.1 and 3.5). The explosion energy of a model is mostly determined by the choice of the initial values of these luminosities (in particular those of $v_{\mathrm{e}}$ and $\bar{v}_{\mathrm{e}}$ ). These initial values are constrained by the prescribed total loss of neutrino energy from the core during the proto-neutron star cooling, $\Delta E_{v, \text { core }}^{\text {tot }}$ and by the total loss of lepton number, $\Delta Y_{\mathrm{e}, \text { core }}$ (see Eqs. (D.13)-(D.16) in Scheck et al. 2006, where $L_{v}^{\mathrm{tot}, 0} t_{\mathrm{L}}$ in the last equation should be replaced by $\Delta E_{v, \text { core }}^{\text {tot }}$. The relative contribution of $v_{\mathrm{e}}$ to the total core luminosity is set to $20 \%$, (i.e., $K_{v_{\mathrm{e}}}=0.2$ in terms of the parameters introduced in Scheck et al. 2006), the contribution of $\bar{v}_{\mathrm{e}}$ is determined from requesting $\Delta Y_{\mathrm{e}, \text { core }}=0.3$, and the muon 


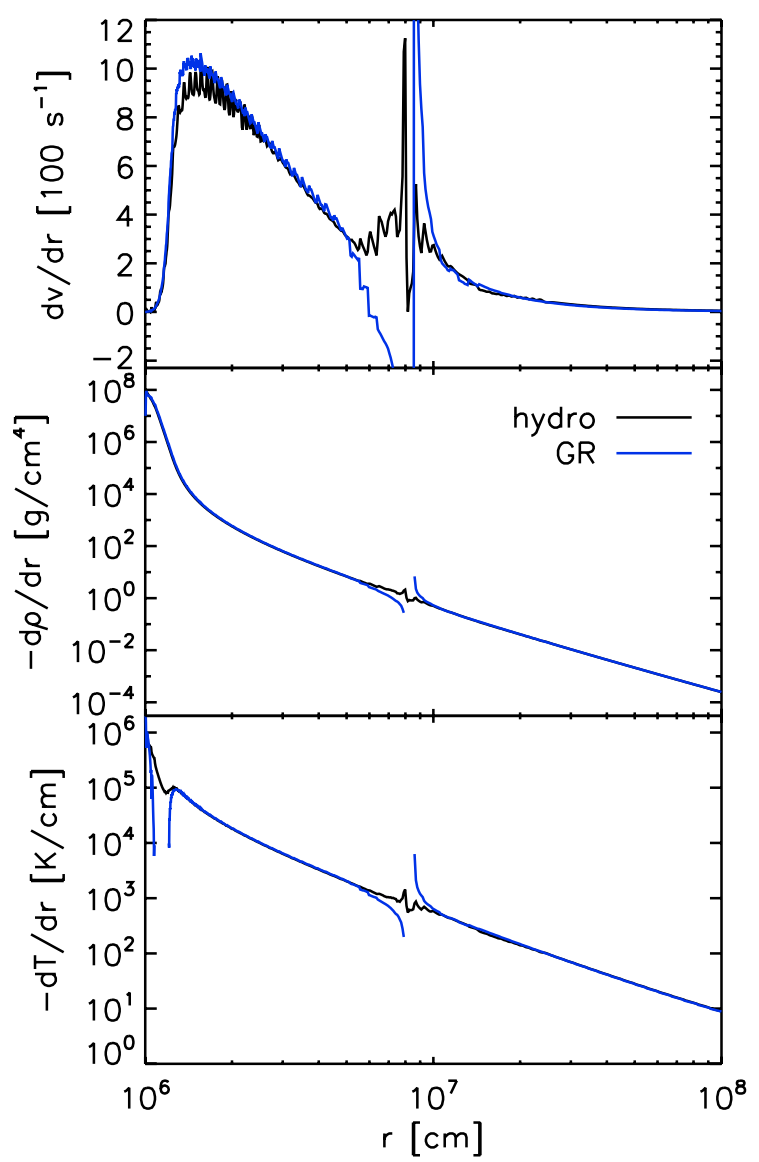

Fig. 1. Derivatives of the velocity (top), density (middle), and temperature (bottom) as functions of radius from our hydrodynamic model M15-11-r1 at $1.5 \mathrm{~s}$ after bounce (black lines) compared to these derivatives as computed from the relativistic stationary wind equations of Eqs. (5)-(7) in Thompson et al. (2001) (blue curves). These equations were evaluated by using the values of all gas quantities as provided by our hydrodynamic model. Consistency between our hydrodynamics results (with approximative treatment of relativity) and the fully relativistic wind solution would require the corresponding lines to lie on top of each other. The agreement is very good and the sonic point is located at about $80 \mathrm{~km}$ in both cases. This location is a critical point of the wind equations, which explains the pathological behavior of the curves there. The evaluation of the expression for the temperature gradient is numerically inaccurate in a region where the two terms in Eq. (7) of Thompson et al.'s paper are very large and have opposite signs, in which case $\mathrm{d} T / \mathrm{d} r$ becomes slightly positive while the hydrodynamical result is still negative.

and tau neutrino contributions then follow from Eq. (D.12) in Scheck et al. (2006). The mean energies of the neutrinos entering the computational grid at the inner boundary are chosen to be $\left\langle\epsilon_{v_{\mathrm{e}}}\right\rangle^{\mathrm{ib}}=12 \mathrm{MeV},\left\langle\epsilon_{\bar{\nu}_{\mathrm{e}}}\right\rangle^{\mathrm{ib}}=16 \mathrm{MeV}$, and $\left\langle\epsilon_{\bar{\gamma}_{x}}\right\rangle^{\mathrm{ib}}=20 \mathrm{MeV}$ when $v_{x}$ denotes muon and tau neutrinos and antineutrinos. These energies are kept constant with time.

Because of the contraction and postbounce accretion of the proto-neutron star, the density and optical depth in the layers near the inner grid boundary can increase to such large values that the application of our transport approximation becomes inefficient by the required very fine zoning, and the equation of state fails to describe the dense stellar matter. Whenever the $v_{\mathrm{e}}$ optical depth begins to exceed a certain value (usually chosen to be 300$)$, we shift the inner boundary to a larger radius $\widetilde{R}_{\mathrm{ib}}\left(t_{\text {cut }}\right)$ and thus to a larger mass shell where the neutrino optical depth is significantly lower (usually 200 for $v_{\mathrm{e}}$ ). The additional excised baryonic mass is added to the previous core mass and the gravitational mass of the new, increased core is set equal to the gravitational mass computed at radius $\widetilde{R}_{\mathrm{ib}}\left(t_{\text {cut }}\right)$ where the new inner grid boundary is placed (see Sect. 1.3). The subsequent motion of the new boundary for $t>t_{\text {cut }}$ is assumed to follow the function

$R_{\mathrm{ib}}^{\prime}(t)=R_{\mathrm{f}}+\left(\widetilde{R}_{\mathrm{ib}}\left(t_{\mathrm{cut}}\right)-R_{\mathrm{f}}\right) \exp \left[v\left(t-t_{\mathrm{cut}}\right) /\left(\widetilde{R}_{\mathrm{ib}}\left(t_{\mathrm{cut}}\right)-R_{\mathrm{f}}\right)\right]$,

where $v<0$ is the recession velocity of the mass shell of the new boundary at time $t_{\text {cut }}$. The new boundary contracts in a very similar way as the previous one because the removed shell is very narrow. The neutrino luminosities and mean energies of the streaming neutrinos imposed at the new boundary at $t=t_{\text {cut }}$ are chosen to be the values computed with the transport scheme at this radius and to have the same time behavior as the initial boundary luminosities and mean energies.

\subsection{Gravity}

Relativistic effects are taken into account in our Newtonian hydrodynamics code by using an "effective relativistic gravitational potential" (Rampp \& Janka 2002). The simulations presented in this paper (different from those of Scheck et al. 2006) employ the improved version of this potential described by Marek et al. (2006), who found excellent agreement with fully relativistic calculations during core collapse and the first several hundred milliseconds after core bounce (tests for the later neutrino-wind phase can be found in Sect. 2).

According to Rampp \& Janka (2002) and Marek et al. (2006), the relativistic equation of motion can be rearranged in a form similar to the Newtonian one by replacing the Newtonian gravitational potential with a modified TOV potential:

$\Phi_{\mathrm{TOV}}(r)=-4 \pi G \int_{r}^{\infty} \frac{\mathrm{d} r^{\prime}}{r^{\prime 2}}\left(\frac{\widetilde{m}_{\mathrm{TOV}}}{4 \pi}+\frac{r^{\prime 3} P}{c^{2}}\right) \frac{1}{\Gamma^{2}}\left(\frac{\rho c^{2}+e+P}{\rho c^{2}}\right)$,

where $\rho$ is the rest-mass density, $e=\rho \epsilon$ the internal energy density with $\epsilon$ being the specific internal energy, and $P$ the gas pressure. The usually rather small corrections of the gravitational potential due to neutrino pressure, energy density, and flux terms (see Marek et al. 2006) are neglected in Eq. (3). The "modified TOV mass" $\widetilde{m}_{\mathrm{TOV}}$ is given by

$\widetilde{m}_{\mathrm{TOV}}(r)=4 \pi \int_{0}^{r} \mathrm{~d} r^{\prime} r^{\prime 2}\left(\rho+\frac{e}{c^{2}}\right) \Gamma$

with the metric function

$\Gamma=\sqrt{1+\frac{v^{2}}{c^{2}}-\frac{2 G \widetilde{m}_{\mathrm{TOV}}}{r c^{2}}}$

The extra factor $\Gamma$ in Eq. (4) compared to the relativistic definition of the TOV mass enters the mass integral for reasons of consistency with the Newtonian hydrodynamics equations and accounts for the fact that in the Newtonian code there is no distinction between local proper volume and coordinate volume, i.e., $\mathrm{d} \mathcal{V}=\mathrm{d} V$ (for more details, see Marek et al. 2006).

There is, however, an important difference of our calculations compared to those performed by Marek et al. (2006). While the latter included the whole neutron star down to the center, the use of the inner grid boundary at a radius $R_{\mathrm{ib}}>0$ in the present work prevents the evaluation of the integral in Eq. (4) within the neutron star core. We solve this problem by starting our calculations with a given value of the modified TOV mass of the core 
at $t=0, \widetilde{m}_{\mathrm{TOV}}\left(R_{\mathrm{ib}}, 0\right)$, which was provided to us as part of the data set for the initial conditions of our simulations. For $t>0$ we then approximately evolve the modified TOV mass according to the expression

$$
\begin{aligned}
\widetilde{m}_{\mathrm{TOV}}\left(R_{\mathrm{ib}}, t\right)=\widetilde{m}_{\mathrm{TOV}}\left(R_{\mathrm{ib}}, 0\right) & -\int_{0}^{t} L_{v}^{\mathrm{ib}}\left(t^{\prime}\right) \mathrm{d} t^{\prime} \\
& -\int_{0}^{t} 4 \pi R_{\mathrm{ib}}^{2}\left(t^{\prime}\right) P_{\mathrm{ib}}\left(t^{\prime}\right) \frac{\mathrm{d} R_{\mathrm{ib}}}{\mathrm{d} t^{\prime}} \mathrm{d} t^{\prime}
\end{aligned}
$$

where the second term on the rhs yields the energy loss from the neutron star core by the total neutrino luminosity at the inner boundary, $L_{v}^{\mathrm{ib}}(t)$, and the last term represents the compression $(P \mathrm{~d} V)$ work done on the core at the contracting inner boundary. The total modified TOV-mass at radius $r$, which we consider as "gravitational mass", is thus given by

$\widetilde{m}_{\mathrm{TOV}}(r, t)=\widetilde{m}_{\mathrm{TOV}}\left(R_{\mathrm{ib}}, t\right)+4 \pi \int_{R_{\mathrm{ib}}}^{r} \mathrm{~d} r^{\prime} r^{\prime 2}\left(\rho+\frac{e}{c^{2}}\right) \Gamma$.

\section{Comparison with fully relativistic wind solutions}

In this section we will discuss our simulation approach in view of other published work on relativistic steady-state solutions for neutrino-driven winds. General relativistic (GR) effects have been recognized to cause important changes of the neutrino wind properties, e.g., to lead to a decrease of the expansion timescale and to an increase of the wind entropy, see Qian \& Woosley (1996), Cardall \& Fuller (1997), Sumiyoshi et al. (2000). A comprehensive discussion of these effects in comparison with the Newtonian treatment was provided by Otsuki et al. (2000) and Thompson et al. (2001).

In our simulations we account for relativistic gravity only by using a modified effective potential (Sect. 1.3), but otherwise we solve the Newtonian equations of hydrodynamics. We also ignore relativistic redshift and ray bending effects in our description of the neutrino transport (cf. Scheck et al. 2006).

The use of the generalized potential in a Newtonian hydrodynamics code was shown previously to yield results in very good agreement with relativistic core-collapse simulations up to several $100 \mathrm{~ms}$ after core bounce (Liebendörfer et al. 2005; Marek et al. 2006). For the later neutrino-wind phase we tried to compare with solutions plotted by Thompson et al. (2001) for cases when our neutron star masses, neutron star radii, and neutrinoheating rates were similar to the ones considered in that paper. Unfortunately, we were unable to find moments in our simulations where all relevant parameters match up exactly the cases considered by Thompson et al. (2001). As far as a comparison was possible, we observed satisfactory agreement in the main properties characterizing the wind.

A more quantitative comparison is hampered by the fact that relativistic neutrino-wind simulations are not available to us. We therefore decided to make use of Eqs. (5)-(7) for the velocity derivative, $\partial v / \partial r$, the density derivative, $\partial \rho / \partial r$, and the temperature derivative, $\partial T / \partial r$, in the paper of Thompson et al. (2001). Figure 1 shows these derivatives as functions of radius at a certain time for one of our models, compared to the results from Thompson et al.'s fully relativistic expressions. Evaluating the latter, we took all quantities on the rhs. of the formulas (velocity $v$, adiabatic sound speed $c_{\mathrm{s}}$, density $\rho$, gravitational mass $M$, neutrino heating rate $\dot{q}$, etc.) from our model. Ideally, the pairs of corresponding curves in Fig. 1 should fall on top of each other, which would demonstrate consistency of both calculations. The overall agreement of the two cases is very good, with a small difference being visible only around the maximum of the acceleration, which, however, is located at the same radius. Also the sonic point is nearly at the same position of about $80 \mathrm{~km}$ (we are not disturbed by the pathological behavior of the curves in this region, where the expressions for the derivatives have a critical point). We therefore conclude that our approach reproduces the most important features of the relativistic solution, and that relativistic kinematics (which we ignore) is of minor importance compared to the effects of the stronger GR potential, which makes the proto-neutron star more compact and the density and temperature gradients in the neutrinospheric region steeper than in Newtonian gravity.

We also compared our neutrino heating and cooling rates with those used by Otsuki et al. (2000) and Thompson et al. (2001). Figure 2 shows the radius-dependent net (i.e., heating minus cooling) specific rate of neutrino energy deposition by the $\beta$-processes according to Eqs. (20) and (21) of Thompson et al. (2001) with and without corrections for relativistic redshift and ray bending, evaluated at all radii with the stellar parameters and the neutrinospheric values of the $v_{\mathrm{e}}$ and $\bar{v}_{\mathrm{e}}$ luminosities and mean energies from one of our simulations. The data were taken from the same model and time used in Fig. 1. The behavior of both curves agrees qualitatively with Fig. 4a of Otsuki et al. (2000). Close to the neutrinosphere ray bending effects enhance the net heating (since GR causes a reduction of the flux factor as visible for Thompson et al.'s prescription of this quantity in Fig. 2), whereas gravitational redshifting of the neutrino luminosities and energies grows monotonically with distance from the neutrinosphere and finally wins, reducing the GR rate below the Newtonian value. Otsuki et al. (2000) performed test calculations to disentangle the influence of GR corrections in the neutrino treatment from that of the relativistic terms in the wind structure equations. In spite of the sizable change of the local heating rate, Otsuki et al. (2000) found that neutrino redshift and ray bending have only little impact on the wind entropy. Similar conclusions were arrived at by Thompson et al. (2001).

In Fig. 2 also the total specific rate of neutrino heating and cooling from our hydrodynamical model is displayed. This rate includes all contributing processes, i.e. besides the $\beta$-reactions of $v_{\mathrm{e}}$ and $\bar{v}_{\mathrm{e}}$ absorption and production also energy transfer by the scattering off electrons, positrons, and free nucleons, and neutrino-antineutrino pair annihilation, to which neutrinos of all flavors contribute (cf. the appendix of Scheck et al. 2006). This total rate is similar to the neutrino capture and emission rates of Eqs. (20) and (21) of Thompson et al. (2001), because for the considered situation the neutrino luminosities are high (see Fig. 2) and therefore the wind mass loss rate is large and the wind entropy fairly low ( $s_{\mathrm{W}} \lesssim 50 k_{\mathrm{B}}$ per nucleon). At such conditions of high wind density and modest abundance of $\mathrm{e}^{+} \mathrm{e}^{-}$-pairs, the other reactions do not contribute significantly to the total rate of energy deposition.

We point out here that our approximative treatment of neutrino transport evolves the transport solution self-consistently with the temperature and density structure of the stellar medium. This is different from the light-bulb approach of previous steadystate or hydrodynamical wind studies (e.g., Sumiyoshi et al. 2000; Otsuki et al. 2000; Thompson et al. 2001). Inside the neutrinosphere neutrinos and matter are in equilibrium, around the neutrinosphere neutrinos begin to decouple thermodynamically from the medium, and at some larger distance they start streaming freely. A changing radial structure of the contracting neutron star leads to changes of the neutrino luminosities and mean energies, and the gradual loss of neutrinos drives the cooling and deleptonization of the surface-near layers of the neutron star. 


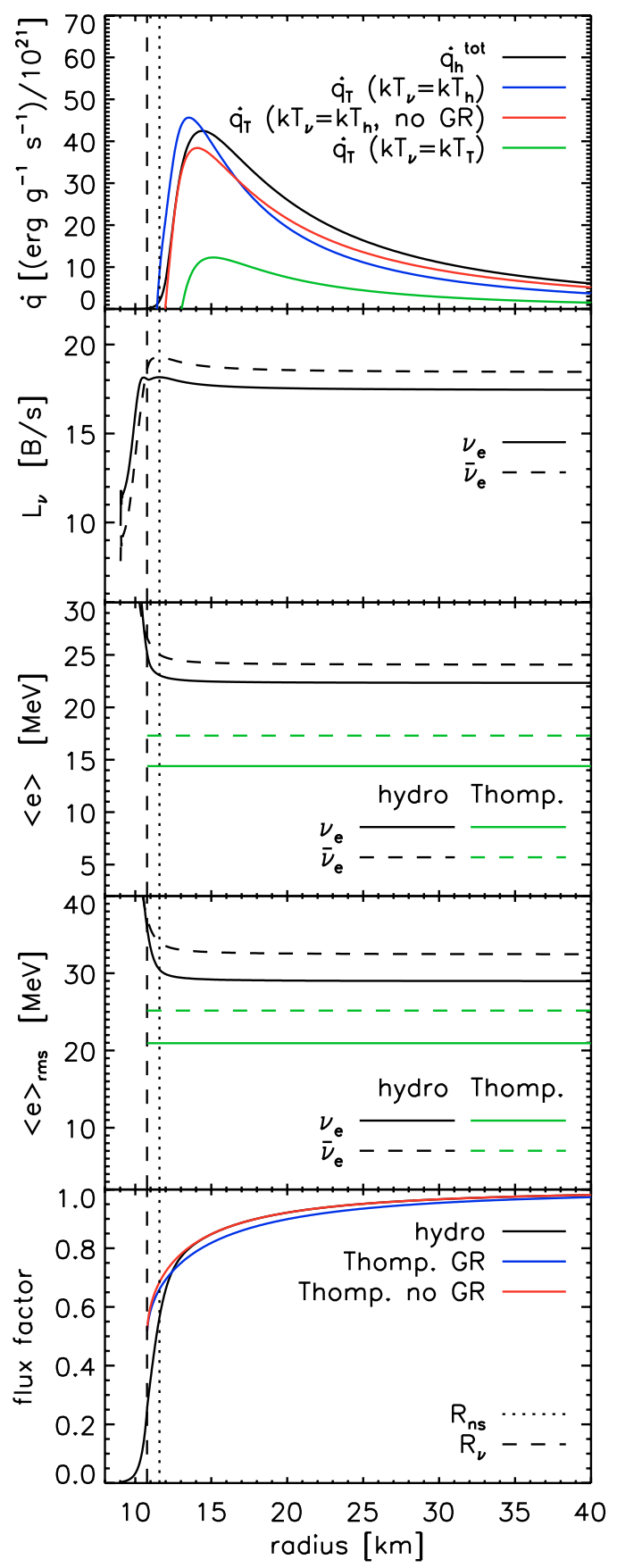

Fig. 2. Radial profiles of the net total neutrino heating rate $\dot{q}^{\text {tot }}$ (top), $v_{\mathrm{e}}$ and $\bar{v}_{\mathrm{e}}$ luminosities $L_{v}$ (second panel, in bethe per second or $10^{51} \mathrm{erg} \mathrm{s}^{-1}$ ), mean neutrino energies $\left\langle\epsilon_{v}\right\rangle$ (third panel; Eq. (8)), rms energies $\left\langle\epsilon_{v}\right\rangle_{\text {rms }}$ (fourth panel; Eq. (9)), and flux factor for model M15-11$\mathrm{r} 1$ at $1.5 \mathrm{~s}$ after bounce. For comparison with Thompson at al. (2001), we also show $\left\langle\epsilon_{v}\right\rangle$ and $\left\langle\epsilon_{v}\right\rangle_{\text {rms }}$ as used in that paper, rescaled to our values of $L_{v_{\mathrm{e}}}$ and $L_{\bar{v}_{\mathrm{e}}}$, the flux factor with and without relativistic corrections from that work, and the Newtonian and GR charged-current heating plus cooling rates using Thompson et al.'s formulas, evaluated with our neutrino luminosities and rms energies (red and blue curves) or with the rescaled rms energies of Thompson et al. (green curve). The vertical dashed line marks the position of the $v_{\mathrm{e}}$-sphere, and the vertical dotted line the neutron star "surface" at a density of $10^{11} \mathrm{~g} \mathrm{~cm}^{-3}$.

In previous wind studies (except full supernova models), such a coupling and interdependence was ignored. Close to the neutron star surface the flux factor (or flux dilution factor) used in our transport, which is based on a Monte Carlo calibration by
Janka (1991), is lower than the vacuum approximation chosen by Thompson et al. (2001) and Otsuki et al. (2000), see Fig. 2. Thompson et al. (2001) have tested the improved description by Janka (1991) and found that its effects are negligible for the range of model conditions considered by them. This, however, is true only during phases where the density gradient near the neutrinosphere is very steep and in regions where the neutrino luminosities have already reached their asymptotic values.

The most important difference of our simulations compared to other relativistic wind studies is the different treatment of the spectra in the neutrino transport. In our "grey" but nonequilibrium description of neutrino number and energy transport, we determine a neutrino spectral temperature that is independent of the matter temperature and can be different from it (for details, see the appendix of Scheck et al. 2006). This leads to higher mean energies of $v_{\mathrm{e}}$ and $\bar{v}_{\mathrm{e}}$ radiated from the neutrinosphere than considered in the other works. Figure 2 shows these mean energies as functions of radius, defined once as the ratio of neutrino energy flux to neutrino number flux,

$\left\langle\epsilon_{v}\right\rangle \equiv \frac{L_{e}}{L_{n}}$,

and another time as rms energy,

$\left\langle\epsilon_{v}\right\rangle_{\mathrm{rms}} \equiv k_{\mathrm{B}} T_{v} \sqrt{\frac{\mathcal{F}_{5}\left(\eta_{v}\right)}{\mathcal{F}_{3}\left(\eta_{v}\right)}}$,

which is the energy which enters the calculation of the neutrino absorption rates on nucleons (cf., for example, Scheck et al. 2006). In Eq. (9), $T_{v}$ and $\eta_{v}$ are the spectral temperature and degeneracy, assuming that the neutrino spectra have Fermi-Dirac shape, in which case

$$
\mathcal{F}_{n}(y)=\int_{0}^{\infty} \mathrm{d} x \frac{x^{n}}{1+\exp (x-y)} .
$$

For comparison, Fig. 2 also presents the corresponding mean energies and rms energies as used by Thompson et al. (2001), appropriately scaled by $L_{v}^{1 / 4}$ to account for the larger neutrino luminosities considered here, and taking $\eta_{v}=0$ for neutrinos and antineutrinos. The net heating rate computed with these rms energies is significantly lower than the heating rate from our hydrodynamic model (Fig. 2).

At first glance, our mean energies for $v_{\mathrm{e}}$ and $\bar{v}_{\mathrm{e}}$ might appear on the large side. One must, however, take into account that the mean energies in our simulations are significantly lower in the first $\sim 0.5$ seconds when the neutron star is still rather extended, and only increase as it heats up during contraction. They reach a maximum between one and two seconds after bounce to decrease afterwards as the proto-neutron star cools (see Sect. 3). So the conditions plotted in Fig. 2 correspond to a time when the neutrino luminosities are still rather high and the mean energies in this phase at their maximum. Moreover, one should remember that we ignore gravitational redshifting in our transport. The redshift from the neutrinosphere at radius $R_{v}$ to infinity after the contraction of the neutron star can become quite significant, $\sqrt{1-2 G M /\left(R_{v} c^{2}\right)}=\sqrt{1-R_{\mathrm{S}} / R_{v}} \approx 0.7 \ldots 0.8$ for $\frac{1}{2} \gtrsim R_{\mathrm{s}} / R_{v} \gtrsim \frac{1}{3}$, which reduces the mean energies for a distant observer by typically 20-30\%. In our Newtonian transport treatment we prefer to use the higher neutrinospheric energies for evaluating the neutrino heating, because the neutrino-wind properties are mostly determined by the heating just outside of the neutrinosphere, where it is also strongest. 
Table 1. Model parameters of our spherically symmetric simulations. The different models are characterized by the chosen contraction of the inner grid boundary, which is expressed in terms of the final radius $R_{\mathrm{f}}$ and the exponential contraction timescale $t_{0}$ (cf. Eq. (1)). Different choices of these values are indiced by the extensions "r1", "r2", etc. of the model names. In addition, different initial luminosities of $v_{\mathrm{e}}$ plus $\bar{v}_{\mathrm{e}}$ (measured in bethe $[\mathrm{B}]=10^{51}$ erg per second) are imposed at the inner grid boundary in case of our standard luminosity behavior (constant until $1 \mathrm{~s}$ and then a $t^{-3 / 2}$ decay). These variations are reflected by the extensions "11", "12", etc. in the model names. Moreover, the timedependence of the boundary luminosity has been modified to a luminosity decay that is more rapid than in the standard description (Eqs. (11) and (12); models with "lt" in their names).

\begin{tabular}{cccc}
\hline \hline Model & $\begin{array}{c}\text { Contraction } \\
\left(R_{\mathrm{f}}, t_{0}\right)\end{array}$ & $\begin{array}{c}L_{v_{\mathrm{e}}}^{\mathrm{ib}}+L_{\overline{\mathrm{v}}_{\mathrm{e}}}^{\mathrm{ib}} \\
{[\mathrm{B} / \mathrm{s}]}\end{array}$ & $\begin{array}{c}\text { Progenitor Mass } \\
{\left[M_{\odot}\right]}\end{array}$ \\
\hline M15-11-r1 & $9 \mathrm{~km} ; 0.1 \mathrm{~s}$ & 52.5 & 15 \\
M15-11-r2 & $9 \mathrm{~km} ; 0.2 \mathrm{~s}$ & 52.5 & 15 \\
M15-11-r5 & $11 \mathrm{~km} ; 0.1 \mathrm{~s}$ & 52.5 & 15 \\
M15-11-r6 & $14 \mathrm{~km} ; 0.1 \mathrm{~s}$ & 52.5 & 15 \\
M15-12-r1 & $9 \mathrm{~km} ; 0.1 \mathrm{~s}$ & 38.6 & 15 \\
M15-13-r3 & $10 \mathrm{~km} ; 0.1 \mathrm{~s}$ & 35.8 & 15 \\
M15-1t2-r3 & $10 \mathrm{~km} ; 0.1 \mathrm{~s}$ & 55.2 & 15 \\
M15-lt1-r4 & $10.5 \mathrm{~km} ; 0.1 \mathrm{~s}$ & 55.8 & 15 \\
M10-11-r1 & $9 \mathrm{~km} ; 0.1 \mathrm{~s}$ & 52.5 & 10 \\
M10-15-r3 & $10 \mathrm{~km} ; 0.1 \mathrm{~s}$ & 30.3 & 10 \\
M20-11-r1 & $9 \mathrm{~km} ; 0.1 \mathrm{~s}$ & 52.5 & 20 \\
M20-13-r3 & $10 \mathrm{~km} ; 0.1 \mathrm{~s}$ & 35.8 & 20 \\
M20-14-r3 & $10 \mathrm{~km} ; 0.1 \mathrm{~s}$ & 33.1 & 20 \\
M25-15-r4 & $10.5 \mathrm{~km} ; 0.1 \mathrm{~s}$ & 30.3 & 25 \\
\hline
\end{tabular}

\section{Results}

In this section we will present the results of our time-dependent hydrodynamic simulations. An overview of the computed models will be given in Sect. 3.1. We will begin with the description of a reference case in Sect. 3.2. This model was computed for a certain choice of the time-dependent contraction of the inner grid boundary and of the neutrino luminosities imposed at this boundary. We will then demonstrate that basic features of the wind termination shock can be understood by simple analytic considerations (Sect. 3.3). Next, we will discuss the neutrino wind evolution in different progenitor stars (Sect. 3.4), and finally will investigate the influence of variations of the conditions at the inner boundary (Sect. 3.5).

\subsection{The computed models}

A list of computed 1D models with their characterizing parameters is given in Table 1 . We have performed simulations for progenitor stars with $10.2 M_{\odot}$ (data provided by A. Heger, personal communication), $15 M_{\odot}$ (model s15s7b2; Woosley \& Weaver 1995), $20 M_{\odot}$ (model s20.0, Woosley et al. 2002), and $25 M_{\odot}$ (model s25a28, Heger et al. 2001). Pre-collapse profiles of the last three models are plotted in the appendix of Buras et al. (2006a). The progenitor cores were computed through core collapse and bounce with the neutrino-hydrodynamics code VERTEX and provided to us as initial conditions for the present studies a few milliseconds after shock formation (A. Marek, personal communication). Extensions of the model names ("r1", "r2",....) indicate different prescriptions for the contraction of the inner grid boundary, whose motion was varied by choosing different values of the final radius $R_{\mathrm{f}}$ and of the exponential contraction timescale $t_{0}$ (Eq. (1)). Larger numbers in this sequence correspond to less quickly contracting or less compact neutron stars. Moreover, we varied the sum of the $v_{\mathrm{e}}$ and $\bar{v}_{\mathrm{e}}$ luminosities imposed at the grid boundary with respect to the initial value as well as time-dependence. In most of the calculations the luminosities were chosen to be constant during the first second of postbounce evolution and to decay proportional to $t^{-3 / 2}$ afterwards as in Scheck et al. (2006). Such models are labelled by the extensions " 11 ", " 12 ", etc., with a higher number meaning a lower initial value of the boundary luminosity. In another set of calculations the boundary luminosities were assumed to have a smoother time-dependence (with no jumps in the time derivative) and in particular with less neutrino energy radiated at late postbounce times. The luminosities were prescribed as

$L(t)= \begin{cases}L_{0}, & \text { if } t \leq 0.5 \mathrm{~s} \\ L_{0} f(t), & \text { if } t>0.5 \mathrm{~s}\end{cases}$

with

$f(t)=\frac{\exp \left[-(t-0.5)^{2}\right]+b\left[1+(t-0.5)^{n}\right]^{-1}}{(1+b)}$,

where the time $t$ is measured in seconds. The corresponding models can be recognized by the letters "lt" in their names. The parameter $n$ was set to 1.5 in both cases, while $b=0.2$ was used for model M15-lt1-r4 and $b=0.3$ for M15-lt2-r3.

A comparison of these models allows us to study the influence of different contraction behavior of the nascent neutron star. The contraction determines the release of gravitational energy from the mantle layers of the compact remnant. The accretion luminosity generated in the mantle adds to the core flux (given by the imposed boundary condition) and has an influence on the explosion timescale and explosion energy of a model and thus on the location of the mass cut and the baryonic mass of the neutron star. The corresponding gravitational mass, which decreases when energy is lost in neutrinos (Eq. (6)), the radius of the neutron star, and the luminosities and mean energies of the radiated neutrinos are crucial parameters that directly affect the neutrinowind properties as functions of time (see Qian \& Woosley 1996).

We note that the supernova models we study here do not permit us to change individually and independently all parameters and conditions that affect the neutrino wind properties and that determine the behavior of the wind termination shock. The wind depends, e.g., on the neutron star gravitational potential and thus on the neutron star mass. The latter becomes larger when the postbounce accretion phase lasts longer and the explosion happens later, or when the progenitor is more massive and therefore the iron core and postbounce accretion rate are larger. More massive progenitors thus tend to produce neutron stars with bigger masses. For this reason one cannot disentangle the influence of the progenitor structure on the wind termination shock from the effects of the neutron star mass on the neutrino wind.

In order to structure the discussion, we decided to first describe basic features in case of a $15 M_{\odot}$ reference model, then to vary the boundary conditions for this model, and finally to present the results for different progenitors.

\subsection{Evolution of a reference case}

In our reference model, M15-11-r1, the conditions at the inner boundary were chosen such that the $15 M_{\odot}$ star explodes with an energy of $\sim 1.5 \times 10^{51} \mathrm{erg}$ and the neutron star attains a final gravitational mass of $\sim 1.2 M_{\odot}\left(1.4 M_{\odot}\right.$ baryonic $)$ and a radius of $10 \mathrm{~km}$ (Table 2).

A mass-shell plot for the space-time evolution of this model is given in Fig. 3. The explosion sets in about $200 \mathrm{~ms}$ after 
Table 2. Results of the $1 \mathrm{D}$ models at the end of the simulations at $t=10 \mathrm{~s}$ after bounce. $M_{\mathrm{bar}}$ is the baryonic mass of the neutron star, $M_{\mathrm{grv}}$ its gravitational mass (Eq. (7)). Both masses are computed for the matter inside the neutron star radius $R_{\mathrm{ns}}$. This radius is defined as the location where the density is $10^{11} \mathrm{~g} \mathrm{~cm}^{-3} . M_{\text {eff }}$ denotes an "effective mass" of the neutron star, for which a Newtonian force equals to the gravitational force associated with the modified TOV potential of Eq. (3) at radius $R_{\mathrm{ns}} . \Delta E_{\mathrm{tot}}$ is the total energy radiated in neutrinos of all flavors (measured in bethe $[\mathrm{B}]=10^{51} \mathrm{erg}$ ), $L_{v_{\mathrm{e}}}$ and $L_{\bar{v}_{\mathrm{e}}}$ are the luminosities of electron neutrinos and antineutrinos measured at $500 \mathrm{~km}$ (without gravitational redshifting), $\left\langle\epsilon_{v_{\mathrm{e}}}\right\rangle$ and $\left\langle\epsilon_{\bar{v}_{\mathrm{e}}}\right\rangle$ are the corresponding mean energies, $E_{\exp }$ is the explosion energy (note that this energy can still decrease somewhat after $10 \mathrm{~s}$ because of the negative binding energy of the outer stellar layers, which is not included in the given numbers), $t_{\mathrm{exp}}$ is the postbounce time when the explosion sets in (defined as the moment when the energy of expanding postshock matter exceeds $10^{49} \mathrm{erg}$ ), $s_{\mathrm{w}}$ is the asymptotic wind entropy per nucleon, and $s_{\mathrm{rs}}$ the entropy of the outflow after its deceleration in the wind termination shock.

\begin{tabular}{|c|c|c|c|c|c|c|c|c|c|c|c|c|c|c|}
\hline Model & $\begin{array}{c}\text { time } \\
{[\mathrm{s}]}\end{array}$ & $\begin{array}{c}M_{\text {bar }} \\
{\left[M_{\odot}\right]}\end{array}$ & $\begin{array}{c}M_{\text {grv }} \\
{\left[M_{\odot}\right]}\end{array}$ & $\begin{array}{c}M_{\text {eff }} \\
{\left[M_{\odot}\right]}\end{array}$ & $\begin{array}{c}\Delta E_{\mathrm{tot}} \\
{[100 \mathrm{~B}]}\end{array}$ & $\begin{array}{c}R_{\mathrm{ns}} \\
{[\mathrm{km}]}\end{array}$ & $\begin{array}{c}L_{v_{\mathrm{e}}} \\
{[\mathrm{B} / \mathrm{s}]}\end{array}$ & $\begin{array}{c}L_{\bar{v}_{\mathrm{e}}} \\
{[\mathrm{B} / \mathrm{s}]}\end{array}$ & $\begin{array}{c}\left\langle\epsilon_{v_{\mathrm{e}}}\right\rangle \\
{[\mathrm{MeV}]}\end{array}$ & $\begin{array}{c}\left\langle\epsilon_{\bar{v}_{\mathrm{e}}}\right\rangle \\
{[\mathrm{MeV}]}\end{array}$ & $\begin{array}{c}E_{\exp } \\
{[\mathrm{B}]}\end{array}$ & $\begin{array}{l}t_{\exp } \\
{[\mathrm{s}]}\end{array}$ & $\begin{array}{c}s_{\text {wind }} \\
{\left[k_{\mathrm{B}} / \text { nuc }\right]}\end{array}$ & $\begin{array}{c}s_{\mathrm{rs}} \\
{\left[k_{\mathrm{B}} / \text { nuc }\right]}\end{array}$ \\
\hline M15-11-r1 & 10.0 & 1.399 & 1.207 & 1.910 & 3.422 & 10.09 & 1.73 & 2.06 & 16.19 & 19.52 & 1.486 & 0.201 & 91.10 & 190.55 \\
\hline M15-11-r2 & 10.0 & 1.446 & 1.263 & 2.031 & 3.335 & 10.27 & 1.96 & 2.28 & 16.37 & 19.50 & 1.174 & 0.341 & 94.19 & 191.29 \\
\hline M15-11-r5 & 10.0 & 1.394 & 1.208 & 1.703 & 3.307 & 12.94 & 1.78 & 2.11 & 14.57 & 17.62 & 1.371 & 0.221 & 71.97 & 131.94 \\
\hline M15-11-r6 & 10.0 & 1.440 & 1.258 & 1.648 & 3.274 & 16.71 & 1.61 & 1.93 & 13.04 & 15.98 & 1.043 & 0.241 & 61.04 & 83.63 \\
\hline M15-12-r1 & 10.0 & 1.473 & 1.280 & 2.116 & 3.451 & 9.94 & 1.74 & 2.12 & 16.46 & 20.30 & 1.019 & 0.381 & 100.14 & 193.14 \\
\hline M15-13-r3 & 10.0 & 1.545 & 1.341 & 2.121 & 3.709 & 11.22 & 1.99 & 2.34 & 15.88 & 18.97 & 0.709 & 0.701 & 92.75 & 155.84 \\
\hline M15-lt2-r3 & 10.0 & 1.397 & 1.253 & 1.906 & 2.602 & 11.28 & 1.33 & 1.54 & 15.04 & 18.12 & 1.239 & 0.221 & 90.19 & 132.57 \\
\hline M15-lt1-r4 & 10.0 & 1.395 & 1.260 & 1.878 & 2.421 & 11.81 & 0.96 & 1.12 & 14.33 & 17.40 & 1.231 & 0.221 & 91.02 & 96.92 \\
\hline M10-11-r1 & 10.0 & 1.314 & 1.132 & 1.721 & 3.251 & 10.22 & 1.63 & 1.96 & 15.85 & 19.06 & 1.247 & 0.321 & 83.48 & 476.93 \\
\hline M10-15-r3 & 10.0 & 1.344 & 1.187 & 1.745 & 2.817 & 11.49 & 1.47 & 1.72 & 14.98 & 18.04 & 0.716 & 0.421 & 80.94 & 353.32 \\
\hline M20-11-r1 & 10.0 & 1.422 & 1.233 & 1.973 & 3.388 & 10.10 & 1.74 & 2.05 & 16.23 & 19.52 & 1.486 & 0.181 & 94.18 & 127.89 \\
\hline M20-13-r3 & 10.0 & 1.595 & 1.411 & 2.310 & 3.383 & 11.10 & 1.60 & 1.90 & 15.66 & 18.79 & 0.375 & 0.761 & 105.10 & - \\
\hline M20-14-r3 & 10.0 & 1.523 & 1.332 & 2.118 & 3.437 & 11.03 & 1.61 & 2.00 & 15.69 & 20.00 & 0.847 & 0.421 & 95.32 & 106.84 \\
\hline M25-15-r4 & 10.0 & 1.971 & 1.657 & 2.944 & 5.924 & 11.56 & 2.95 & 3.58 & 16.88 & 20.01 & 1.700 & 0.401 & 113.75 & 117.89 \\
\hline
\end{tabular}

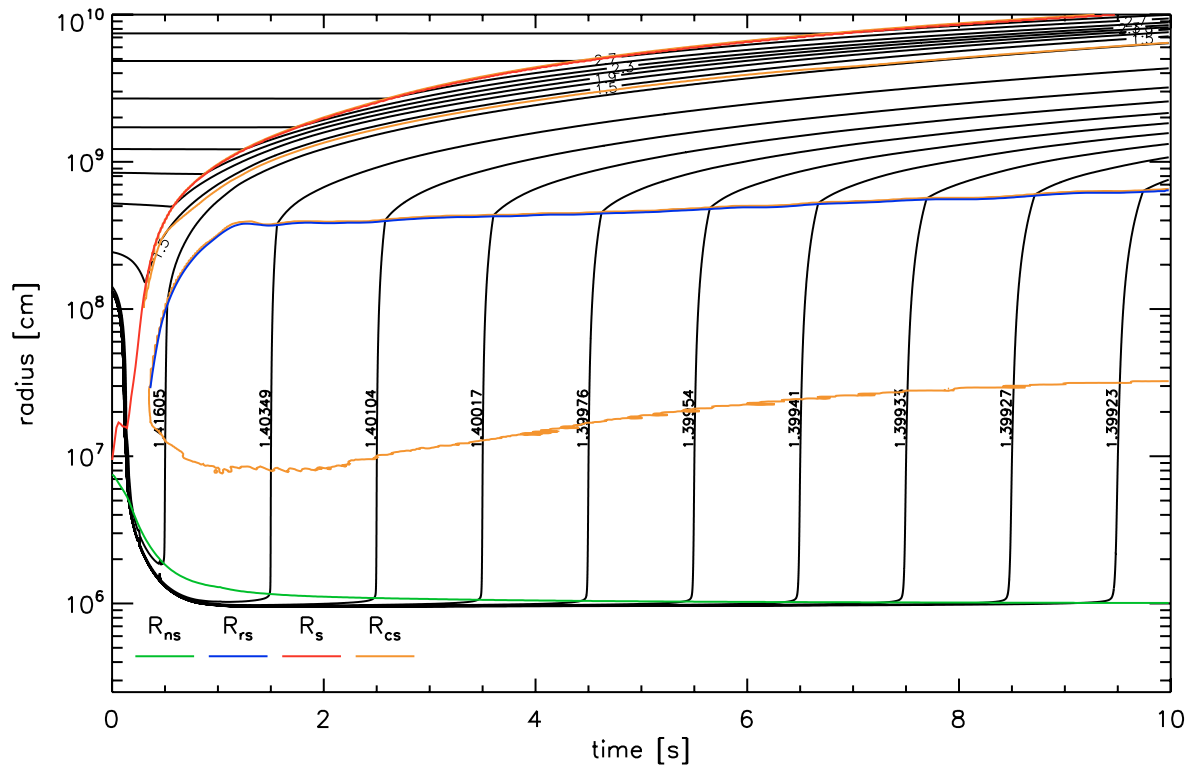

Fig. 3. Mass shell plot for the evolution of model M15-11-r1. The explosion occurs about 0.2 seconds after bounce. The red line marks the supernova shock, the blue line the wind termination shock, the orange lines the locations where the expansion velocity of the gas equals the local sound speed (sonic points), and the green line the neutron star radius defined as the location where the density drops below $10^{11} \mathrm{~g} \mathrm{~cm}^{-3}$. A contact discontinuity separates the dense shell of ejecta that were accelerated by the outgoing shock from the very dilute neutrino-driven wind. Mass shells in the wind are labelled by the corresponding enclosed baryonic masses. bounce (at the time $t_{\exp }$ given in Table 2, which is defined as the moment when the total energy of expanding matter starts to exceed $10^{49} \mathrm{erg}$ ). At this time the stalled shock is revived by neutrino heating and starts continuous expansion with an average velocity of roughly $10000 \mathrm{~km} \mathrm{~s}^{-1}$. On its way out the shock reverses the infall of the swept-up matter. After the onset of the explosion, ongoing neutrino energy transfer drives an outward acceleration of heated material in the gain layer around the neutron star. At the interface between this dilute neutrino-driven wind and the denser outer ejecta a contact discontinuity is formed. Even farther behind the forward shock, the neutrino-driven wind, whose velocity increases rapidly with distance from the neutron star, collides with more slowly moving material and is decelerated again. The strongly negative velocity gradient at this location steepens into a reverse shock when the wind velocity begins to exceed the local sound speed (Janka \& Müller 1995). First indications of a forming wind termination shock can be seen in Fig. 3 at $t \gtrsim 350 \mathrm{~ms}$ post bounce at a radius $r \sim 300 \mathrm{~km}$.

Figure 4 displays the $v_{\mathrm{e}}$ and $\bar{v}_{\mathrm{e}}$ luminosities and the mean energies emitted by the nascent neutron star (gravitational redshift effects are ignored). One can see the accretion phase with its production of accretion luminosity ending at the time the explosion sets in. The following plateau phase until $t \approx 1 \mathrm{~s}$ and subsequent decay of the luminosities show the influence of the time-dependence of the imposed boundary fluxes. This is also the case for the mean neutrino energies. Their values increase during the first second of postbounce evolution because the inner grid boundary and the neutron star radius contract (Fig. 3). 

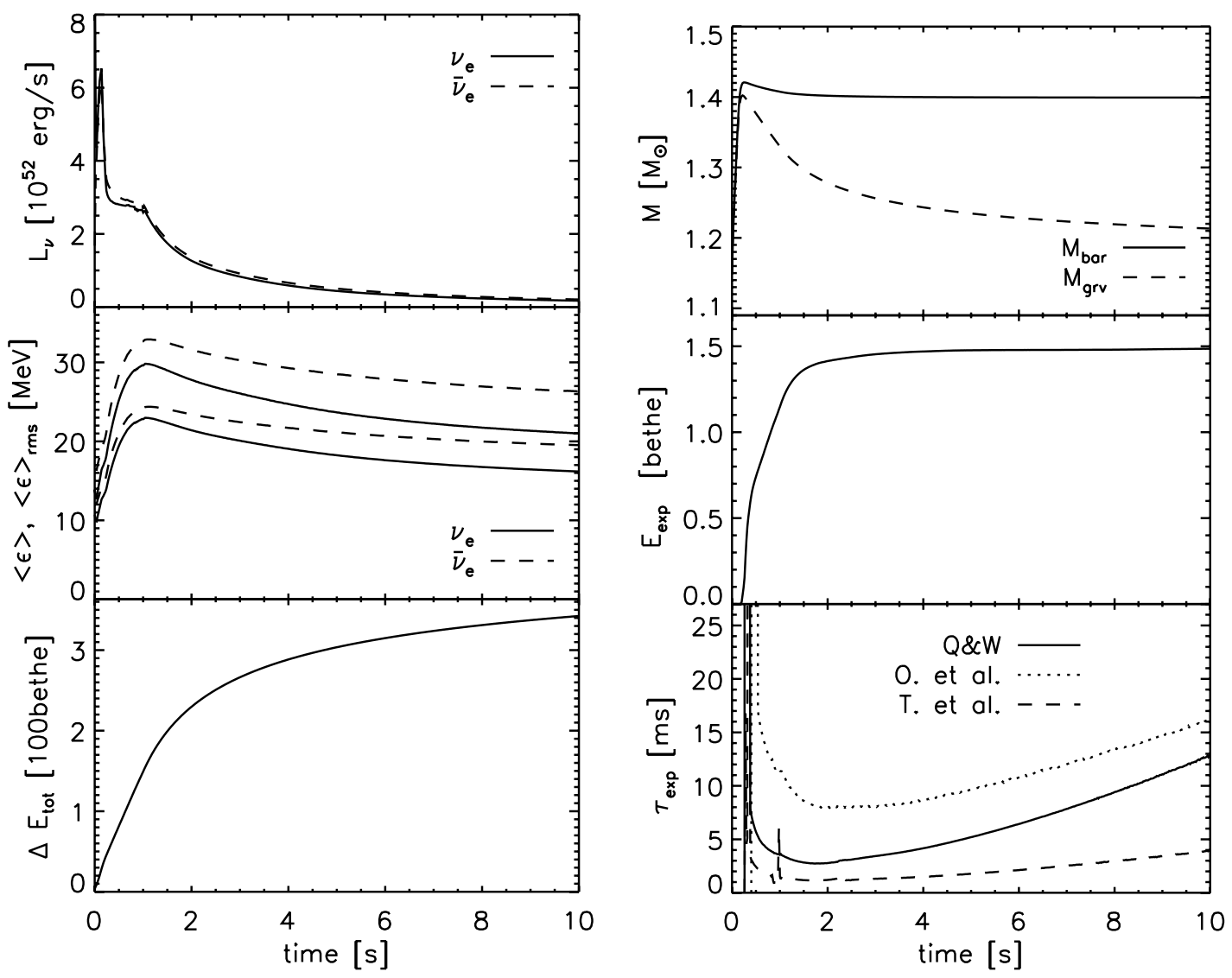

Fig. 4. Luminosities, mean energies according to Eq. (8), and rms energies (Eq. (9), $\left.\left.\langle\epsilon\rangle_{\mathrm{rms}}\right\rangle\langle\epsilon\rangle\right)$ of $v_{\mathrm{e}}$ and $\bar{v}_{\mathrm{e}}$, and total energy radiated in neutrinos of all flavors for model M15-11-r1 as functions of time (left), measured outside of the nascent neutron star (at a radius of $500 \mathrm{~km}$ ). Note that we do not include gravitational redshifting in our neutrino treatment. The rapid decline of the luminosities after about 0.2 marks the end of the accretion phase of the forming neutron star at the onset of the explosion. The panels on the rhs side give the baryonic mass and the gravitational mass (Eq. (7)) of the neutron star in model M15-11-r1, the explosion energy, and the expansion timescales of the neutrino-driven wind as functions of time. For the latter, the results from three different definitions are displayed, namely those used by Qian \& Woosley (1996), Otsuki et al. (2000), and Thompson et al. (2001), given in Eqs. (13), (14), and (15), respectively.

Consequently, the outer layers of the neutron star heat up due to the conversion of gravitational energy to internal energy by compression. After one second the rapid contraction is over and the decay of the boundary luminosities leads to less energy transport into these layers, which therefore begin to cool down, causing the mean energies of the radiated neutrinos to decline.

Figure 4 also provides information about the total energy carried away by neutrinos and antineutrinos of all three lepton flavors, $\Delta E_{\text {tot }}$, as a function of time, and the corresponding reduction of the gravitational mass of the nascent neutron star. The latter is taken to be the modified TOV mass (Eq. (7)) at the neutron star radius $R_{\mathrm{ns}}$ (which is defined as the radius where the density is $10^{11} \mathrm{~g} \mathrm{~cm}^{-3}$ ). In contrast, the baryonic mass of the neutron star, given by the rest mass enclosed by the radius $R_{\mathrm{ns}}$, initially increases in the course of accretion. After the explosion has been lauched, it decreases again only slightly due to the mass loss in the neutrino-driven wind.

The middle panel on the right side of Fig. 4 reveals that only about $50 \%$ of the explosion energy (defined as the integral of the total internal, kinetic, and gravitational energies in all zones where the sum of these energies is positive) are carried by the neutrino-heated shell of matter expanding right behind the shock after the onset of the explosion. The rest is contributed by the early neutrino wind, and after $2 \mathrm{~s}$ the energy has reached $95 \%$ of its final value. The panel below gives the expansion timescales of the ejected mass shells in the neutrino wind. The first definition follows Qian \& Woosley (1996, Eq. (60)), who introduced the dynamical timescale as

$\tau_{\text {dyn }}=\left.\frac{r}{v}\right|_{k_{\mathrm{B}} T=0.5 \mathrm{MeV}}$.

We compare this with the cooling timescale used by Otsuki et al. (2000, Eq. (23)),

$\tau_{T}=\int_{k_{\mathrm{B}} T=0.5 \mathrm{MeV}}^{k_{\mathrm{B}} T=0.5 \mathrm{MeV} / \mathrm{e}} \frac{\mathrm{d} r}{v}$,

variations of which were considered by Witti et al. (1994, cooling time between $T=7 \times 10^{9} \mathrm{~K}$ and $\left.T=3 \times 10^{9} \mathrm{~K}\right)$ and Wanajo et al. (2001, cooling time between $k_{\mathrm{B}} T=0.5 \mathrm{MeV}$ and $\left.k_{\mathrm{B}} T=0.2 \mathrm{MeV}\right)$. The third definition we consider is the one of Thompson et al. (2001, Eq. (32)), who employed the e-folding time of the density instead of that of the temperature,

$\tau_{\rho}=\frac{1}{v}\left|\frac{1}{\rho} \frac{\partial \rho}{\partial r}\right|_{k_{\mathrm{B}} T=0.5 \mathrm{MeV}}^{-1}$,

where we set for our Newtonian simulations $y=1$ in Thompson et al.'s Eq. (32). As can be expected from the fact that the wind is radiation-dominated and therefore $s \propto T^{3} / \rho \sim$ const., the timescale $\tau_{\rho}$ is always significantly shorter than the cooling 

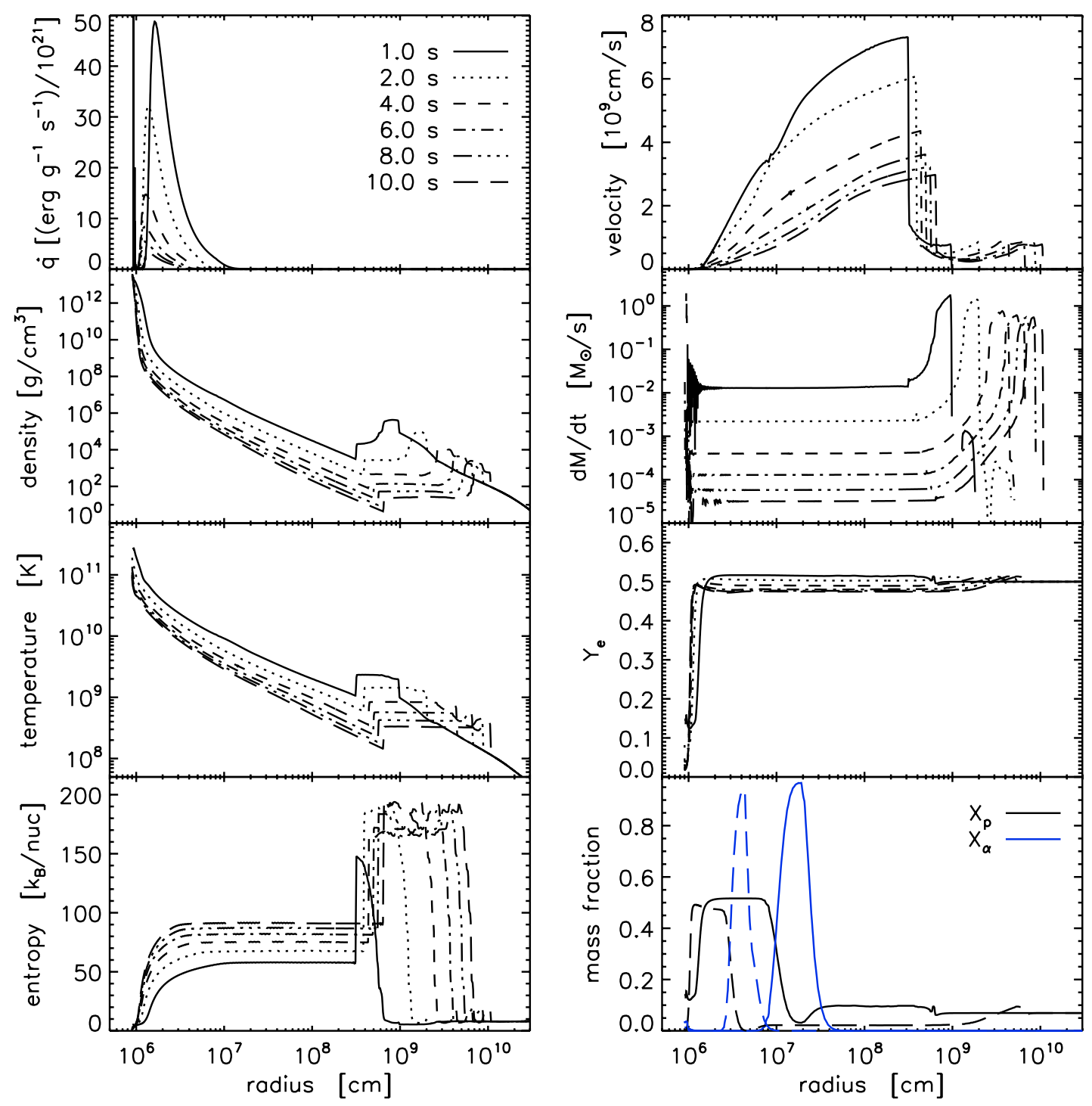

Fig. 5. Radial profiles of the net neutrino-heating rate $\dot{q}$, density, temperature, entropy (left, from top to bottom), velocity, mass loss rate, electron fraction $Y_{\mathrm{e}}$, and mass fractions of free protons and $\alpha$ particles for the neutrino wind in model M15-11-r1 at different postbounce times. For the mass fractions only the information for the first and last moments of time is provided. The wind termination shock is clearly visible in its effects on the velocity, density, temperature, and entropy of the outflow.

timescale $\tau_{T}$ (Fig. 4). Ideally, in such a situation one would expect $\tau_{T} / \tau_{\rho}=3$, which is more closely reached at later stages when the wind entropy is higher (Fig. 5). Due to the different mathematical expressions in Eqs. (14) and (15), the factor 3 is never exactly realized. The third timescale, Eq. (13), yields a result that is between the other two values during most of the computed postbounce evolution and comes closer to the timescale of Eq. (14) in the late stages of the simulations.

In Fig. 5 the radial profiles of different wind quantities are given for our reference model M15-11-r1 at a number of postbounce times. The neutrino heating accelerates the wind to a peak velocity of about $25 \%$ of the speed of light for neutrino luminosities $L_{v_{\mathrm{e}}} \approx L_{\bar{v}_{\mathrm{e}}} \approx 3 \times 10^{52} \mathrm{erg} \mathrm{s}^{-1}$. The maximum velocity decreases as does the heating rate when the luminosities decline with time. The density and temperature in the wind region follow roughly the usual $r^{-3}$ and $r^{-1}$ behavior, respectively, in the region where the entropy is a constant. The profiles are slightly steeper and thus closer to these power laws at later times when the wind entropy is higher and the wind therefore more dominated by radiation pressure. The radial profiles of $\rho$ and $T$ also steepen at larger distance from the neutron star, leading to a visible increase of the wind acceleration at the point where free nucleons recombine to $\alpha$-particles and the neutrino heating ceases (see the corresponding panels in Fig. 5). At this radius the entropy of the outflow reaches its final value. This asymptotic wind entropy increases from about $60 k_{\mathrm{B}}$ per nucleon at $1 \mathrm{~s}$ to around $90 k_{\mathrm{B}}$ at $10 \mathrm{~s}$.

For (approximately) the same values of the $v_{\mathrm{e}}$ and $\bar{v}_{\mathrm{e}}$ luminosities, model M15-11-r1 tends to yield somewhat lower expansion timescales, slightly lower entropies, and a bit higher mass loss rates than those found by Thompson et al. (2001), see Figs. 5, 8, and Tables 1 and 2 there. This can be understood on the one hand by the smaller gravitational mass of the neutron star in our model compared to the canonical $1.4 M_{\odot}$ star considered by Thompson et al. (2001), and on the other hand it is caused by our larger heating rates due to the higher mean neutrino energies (cf. our discussion in Sect. 2). These differences affect the characteristic wind parameters with different sensitivity. According 

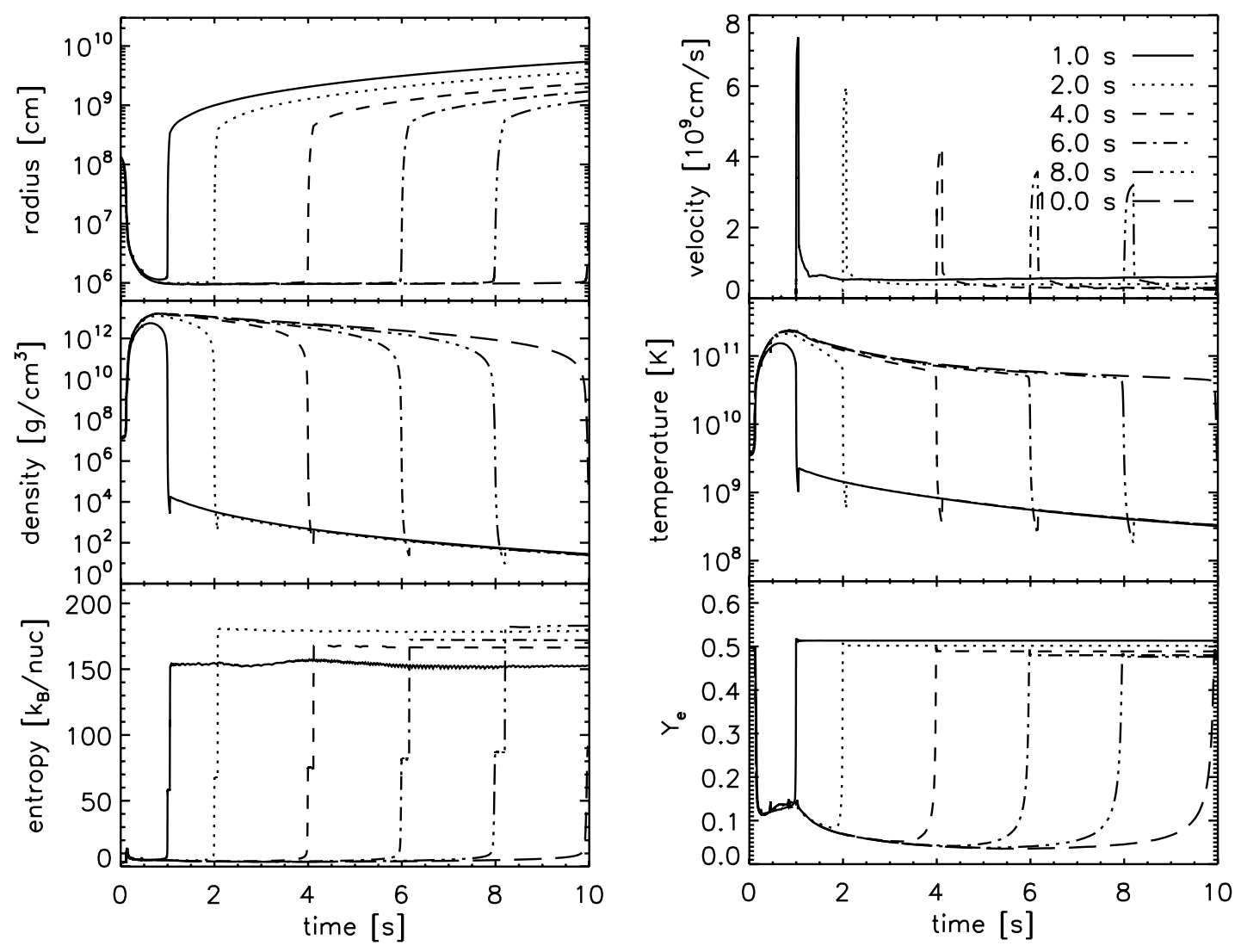

Fig. 6. Radius, density, entropy (left, from top to bottom), velocity, temperature, and electron fraction $Y_{\mathrm{e}}$ as functions of time along the trajectories of different mass shells that are ejected in the neutrino-driven wind of model M15-11-r1. The times correspond to the moments when the mass shells cross a radius of $100 \mathrm{~km}$. After a very rapid expansion, the wind is abruptly decelerated by the termination shock. This leads to an increase of the entropy by more than a factor of two, and to a subsequently much slower decline of the temperature and density.

to Qian \& Woosley (1996), the entropy scales with the neutrino luminosity $L$, the mean neutrino energy $\epsilon$, the neutron star radius $R$, and the neutron star mass $M$ like

$s \propto L^{-1 / 6} \epsilon^{-1 / 3} R^{-2 / 3} M$,

the expansion timescale like

$\tau \propto L^{-1} \epsilon^{-2} R M$,

and the wind mass loss rate like

$\dot{M} \propto L^{5 / 3} \epsilon^{10 / 3} R^{5 / 3} M^{-2}$

(modifications of these relations due to relativistic effects were addressed by Thompson et al. 2001).

During the first $\sim 2 \mathrm{~s}$ after the onset of the explosion, the neutrino wind is p-rich, i.e. $Y_{\mathrm{e}}>0.5$. This is in agreement with explosion models that employ a Boltzmann solver for the spectral neutrino transport (see Buras et al. 2006b; Pruet et al. 2005). Afterwards the electron fraction drops below 0.5 , and gradually the wind develops increasing neutron excess. Qualitatively, this trend to lower $Y_{\mathrm{e}}$ at later times is reproduced when the neutrino luminosities and mean energies from the simulation are inserted into the simple analytic relation $Y_{\mathrm{e}} \sim\left[1+\left(L_{\bar{v}_{\mathrm{e}}} \epsilon_{\overline{\mathrm{e}}_{\mathrm{e}}} / L_{v_{\mathrm{e}}} \epsilon_{\mathrm{v}_{\mathrm{e}}}\right)\right]^{-1}$, although the values do not agree quantitatively. We emphasize here that the gray and approximative treatment of the neutrino transport employed in this work (for a critical assessment, see Scheck et al. 2006) is also not able to yield reliable results for the electron fraction in terms of absolute numbers. The competition of $v_{\mathrm{e}}$ and $\bar{v}_{\mathrm{e}}$ absorption on free neutrons and protons sensitively determines the asymptotic value of $Y_{\mathrm{e}}$, an accurate calculation of which requires detailed information of the neutrino and antineutrino spectra in the comoving frame of the expanding wind matter. The wind at late times might therefore become significantly more neutron rich than predicted in our models. Figure 5 also reveals that the mass loss rate reaches its asymptotic value closest to the neutrinosphere, and only then $Y_{\mathrm{e}}$ and finally the entropy reach their asymptotic values.

At a radius of a few $1000 \mathrm{~km}$, the supersonic wind is abruptly decelerated down in the termination shock. The compression leads to a density and temperature increase. The conversion of kinetic to internal energy in the shock boosts the entropy to more than twice the wind entropy in model M15-11-r1. This is a much more extreme impact of the termination shock than previously suggested in the literature (Thompson et al. 2001). The decelerated wind material is accumulated in a dense shell between the forward and reverse shocks. The pressure across this dense shell is nearly uniform, while the contact discontinuity between the accumulated wind matter and the dense layer of shock-accelerated progenitor gas is clearly visible in the density profiles. One should also notice that the conditions at the wind termination shock are by no means time-independent as previously assumed in nucleosynthesis calculations (e.g., Wanajo et al. 2002). Temperature and density at the reverse shock in model M15-11-r1 evolve, because the radial position of the reverse shock as well as the wind properties change with time. The impact of the wind termination shock on the conditions in the expanding wind mass shells is better visible in Fig. 6, where the time-evolution of different quantities is depicted as 
seen comoving with some selected mass shells. The extremely rapid decline of the temperature and density in the fast wind are stopped and switch over to a much slower evolution. After the wind material has been added to the dense shell between the two shocks, it moves with nearly constant velocity. Its density therefore decays approximately like $\rho \propto t^{-2}$ and because the gas is radiation-dominated, its temperature follows roughly the power law $T \propto t^{-2 / 3}$.

\subsection{Analytic discussion of the wind termination shock}

The behavior of the wind termination shock and its effects on the neutrino-driven outflow can basically be understood by simple analytic considerations. For this purpose we consider the three Rankine-Hugoniot shock jump conditions for mass, momentum and energy flow,

$\rho_{\mathrm{rs}} u_{\mathrm{rs}}=\rho_{\mathrm{w}} u_{\mathrm{w}}$

$P_{\mathrm{rs}}+\rho_{\mathrm{rs}} u_{\mathrm{rs}}^{2}=P_{\mathrm{w}}+\rho_{\mathrm{w}} u_{\mathrm{w}}^{2}$,

$\frac{1}{2} u_{\mathrm{rs}}^{2}+\omega_{\mathrm{rs}}=\frac{1}{2} u_{\mathrm{w}}^{2}+\omega_{\mathrm{w}}$

where the indices $\mathrm{w}$ and rs denote quantities of the wind just ahead of the shock and of the shocked matter just behind the shock, respectively. The fluid velocities $u_{\mathrm{w}}$ and $u_{\mathrm{rs}}$ are measured relative to the shock velocity, $u=v-U_{\mathrm{s}}, P$ is the pressure, $\rho$ the mass density, and $\omega=(\varepsilon+P) / \rho$ the enthalpy per mass unit when $\varepsilon$ is the internal energy density of the gas. In case of radiation-dominated and nondegenerate conditions, one can write $s=(\varepsilon+P) /\left(n_{\mathrm{B}} k_{\mathrm{B}} T\right)$ for the dimensionless entropy normalized by the baryon density $n_{\mathrm{B}} \rho / m_{\mathrm{B}}\left(m_{\mathrm{B}}\right.$ is the average baryon mass), and therefore one gets

$s_{\mathrm{rs}} k_{\mathrm{B}} T_{\mathrm{rs}}-s_{\mathrm{w}} k_{\mathrm{B}} T_{\mathrm{w}}=\frac{1}{2} m_{\mathrm{B}}\left(u_{\mathrm{w}}^{2}-u_{\mathrm{rs}}^{2}\right)$.

Since the wind termination shock strongly decelerates the wind, the postshock and preshock velocities fulfill the relation $u_{\mathrm{w}}^{2} \gg$ $u_{\mathrm{rs}}^{2}$. Thus the postshock entropy is approximately given by

$s_{\mathrm{rs}} \approx s_{\mathrm{W}} \frac{k_{\mathrm{B}} T_{\mathrm{w}}}{k_{\mathrm{B}} T_{\mathrm{rs}}}+\frac{1}{2} \frac{m_{\mathrm{B}} u_{\mathrm{w}}^{2}}{k_{\mathrm{B}} T_{\mathrm{rs}}}$.

Again making the assumption that the gas on both sides of the shock is radiation dominated, the dimensionless entropy per nucleon is given by

$s=f_{\gamma} a_{\gamma} \frac{\left(k_{\mathrm{B}} T\right)^{3}}{n_{\mathrm{B}}}$,

where $a_{\gamma}=a / k_{\mathrm{B}}^{4}=2.08 \times 10^{49} \mathrm{erg}^{-3} \mathrm{~cm}^{-3}$ is related to the radiation constant $a$, and $f_{\gamma}$ is a factor whose exact value depends on the temperature and thus the mixture of radiation and $\mathrm{e}^{+} \mathrm{e}^{-}$-pairs; assuming zero electron degeneracy, the corresponding range of values is $\frac{4}{3} \leq f_{\gamma} \leq \frac{11}{3}$. Equation (24) can be used to express $k_{\mathrm{B}} T$ ahead of and behind the shock in terms of $s$ and $\rho$. Using also that the densities are connected by $\rho_{\mathrm{rs}}=\beta \rho_{\mathrm{w}}$ with $\beta \sim 7$ for a strong shock and radiation-dominated conditions, one derives

$s_{\mathrm{rs}} \approx\left[\frac{s_{\mathrm{w}}^{4 / 3}}{\beta^{1 / 3}}+\alpha^{1 / 3} \frac{u_{\mathrm{w}}^{2}}{\rho_{\mathrm{w}}^{1 / 3}}\right]^{3 / 4} \approx\left[\frac{s_{\mathrm{w}}^{4 / 3}}{\beta^{1 / 3}}+33.5 \frac{u_{\mathrm{w}, 9}^{2}}{\rho_{\mathrm{w}, 2}^{1 / 3}}\right]^{3 / 4}$,

where $\alpha \equiv f_{\gamma} a_{\gamma} m_{\mathrm{B}}^{4} /(8 \beta), u_{\mathrm{w}, 9}$ is the wind velocity measured in $10^{9} \mathrm{~cm} \mathrm{~s}^{-1}, \rho_{\mathrm{w}, 2}$ the wind density in $100 \mathrm{~g} \mathrm{~cm}^{-3}$, and the numerical value in the second expression was calculated with $\beta=7$ and $f_{\gamma}=\frac{4}{3}$. Equation (25) can be rewritten in terms of the wind mass loss rate $\dot{M}_{\mathrm{w}}$ and reverse shock radius $R_{\mathrm{rs}}$, using

$\dot{M}_{\mathrm{w}}=4 \pi R_{\mathrm{rs}}^{2} \rho_{\mathrm{w}} v_{\mathrm{w}}$

and assuming that the shock velocity is negligible, and therefore $v_{\mathrm{w}}=u_{\mathrm{w}}$, which gives

$$
\begin{aligned}
s_{\mathrm{rs}} & \approx\left[\frac{s_{\mathrm{w}}^{4 / 3}}{\beta^{1 / 3}}+(4 \pi \alpha)^{1 / 3} \frac{R_{\mathrm{rs}}^{2 / 3} u_{\mathrm{w}}^{7 / 3}}{\dot{M}_{\mathrm{w}}^{1 / 3}}\right]^{3 / 4} \\
& \approx\left[\frac{s_{\mathrm{w}}^{4 / 3}}{\beta^{1 / 3}}+28.7 \frac{R_{\mathrm{rs}, 8}^{2 / 3} u_{\mathrm{w}, 9}^{7 / 3}}{\dot{M}_{\mathrm{w},-5}^{1 / 3}}\right]^{3 / 4} .
\end{aligned}
$$

Here $R_{\mathrm{rs}, 8}$ is in units of $10^{8} \mathrm{~cm}$ and $\dot{M}_{\mathrm{w},-5}$ is normalized to $10^{-5} M_{\odot}$. If the wind entropy is low, $s_{\mathrm{W}} \ll s_{\mathrm{rs}}$, only the second terms in Eqs. (25) and (27) are relevant.

It is also possible to obtain an estimate of the reverse shock position from known supernova and wind parameters. In case of a strong shock, i.e., $P_{\mathrm{rs}} \gg P_{\mathrm{w}}$, one can derive from Eqs. (19) and (20) the relation $P_{\mathrm{rs}} \approx\left(1-\beta^{-1}\right) \rho_{\mathrm{w}} u_{\mathrm{w}}^{2}$. Using again Eq. (26) for $\rho_{\mathrm{w}}$, one gets

$R_{\mathrm{rs}} \approx \sqrt{\left(1-\frac{1}{\beta}\right) \frac{\dot{M}_{\mathrm{w}} u_{\mathrm{w}}}{4 \pi P_{\mathrm{rs}}}}$.

Assuming the spherical shell between the forward shock at radius $R_{\mathrm{S}} \gg R_{\mathrm{rs}}$ and the reverse shock to have constant pressure and to be radiation dominated, one can make the approximation

$P_{\mathrm{rs}} \sim \frac{f_{\mathrm{exp}} E_{\mathrm{exp}}}{4 \pi R_{\mathrm{s}}^{3}}$,

where $f_{\exp }$ is the fraction of the supernova explosion energy $E_{\exp }$ that is present as internal energy of the gas between forward and reverse shock. Plugging Eq. (29) into Eq. (28) one obtains

$$
\begin{aligned}
R_{\mathrm{rs}} & \sim \sqrt{\left(1-\frac{1}{\beta}\right) \frac{\dot{M}_{\mathrm{w}} u_{\mathrm{w}} R_{\mathrm{s}}^{3}}{f_{\exp } E_{\exp }}} \\
& \approx 4.14 \times 10^{3} \sqrt{\frac{\dot{M}_{\mathrm{w},-5} u_{\mathrm{w}, 9} R_{\mathrm{s}, 10}^{3}}{f_{\exp , 0.1} E_{\mathrm{exp}, 51}}}[\mathrm{~km}] .
\end{aligned}
$$

The numerical value was computed by taking $\beta=7$ and normalizing the shock radius to $10^{10} \mathrm{~cm}$, the explosion energy to $10^{51} \mathrm{erg}$, and the parameter $f_{\exp }$ to 0.1 .

Equations (25) and (27), evaluated with the numbers for the wind quantities from our numerical model, describe the entropy jump at the reverse shock in the simulations very well. The same is true for the reverse shock radius computed from Eq. (28) with the pressure behind the reverse shock, $P_{\mathrm{rs}}$, taken from the hydrodynamic simulations. Equation (30), however, does not yield a satisfactory agreement and for some models fails even qualitatively to reproduce the behavior of the reverse shock radius as a function of time. The reason for this mismatch between simulations and analytic approximation is mainly the fact that the factor $f_{\exp }$ cannot be considered as a constant. Instead, during the expansion of the supernova ejecta, $p \mathrm{~d} V$ work converts internal energy to kinetic energy of the matter swept up by the outgoing shock. Therefore, as time goes on, a smaller and smaller fraction of the explosion energy remains stored as internal energy in the layer between forward and reverse shock. As a consequence, $f_{\exp }$ decreases during the simulated evolution. In the first four seconds, $f_{\exp } \approx 1$ turns out to be a good choice, but lateron $f_{\exp }$ drops monotonically to $f_{\exp } \approx 0.25$ at ten seconds. Taking this into account, Eq. (30) also yields a good description of the reverse shock radius as a function of time. 


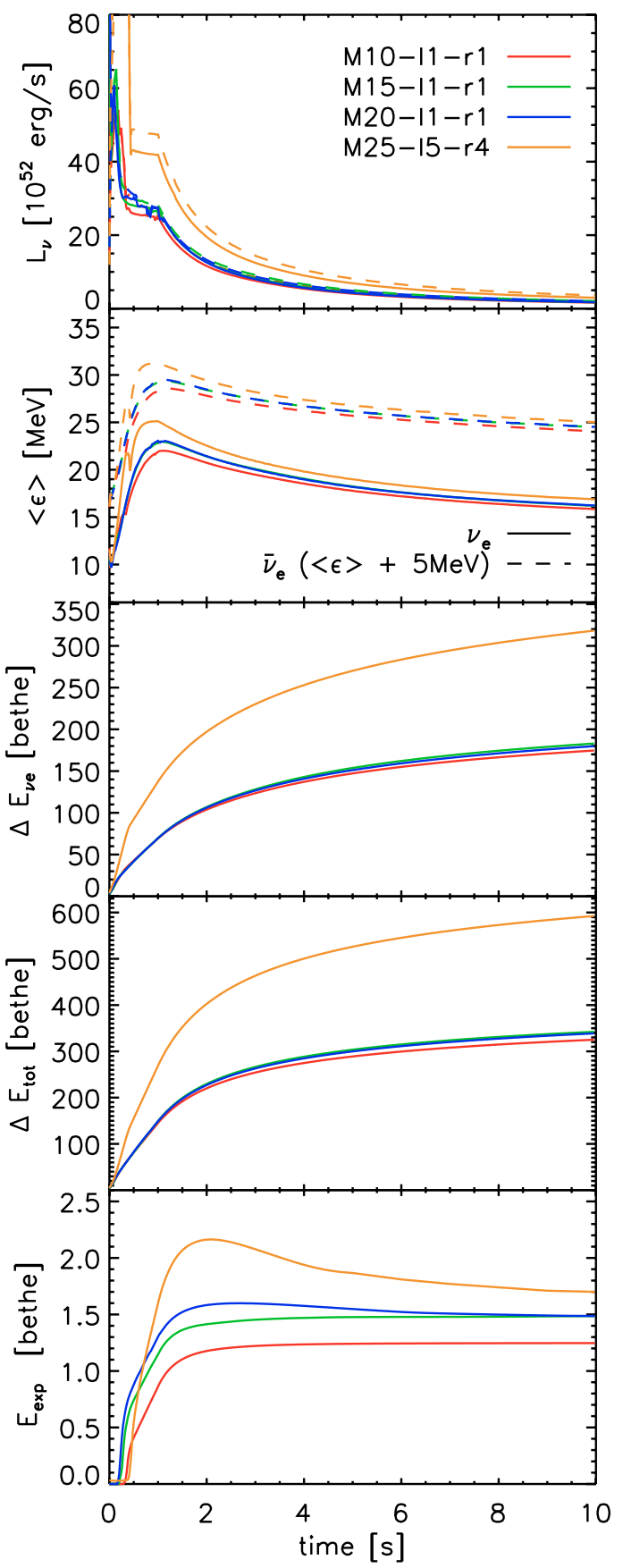

Fig. 7. For a set of simulations with different progenitor stars, M1011-r1, M15-11-r1, M20-11-r1, and M25-15-r4, the different panels show as functions of time (from top to bottom): the radiated luminosities of $v_{\mathrm{e}}$ and $\bar{v}_{\mathrm{e}}$, the mean energies and rms energies of these neutrinos (all measured at a distance of $500 \mathrm{~km}$, disregarding gravitational redshift effects), the cumulative energy emitted in $v_{\mathrm{e}}$ and $\bar{v}_{\mathrm{e}}, \Delta E_{v_{\mathrm{e}}}$, the total energy released in neutrinos and antineutrinos of all flavors, $\Delta E_{\mathrm{tot}}$, and the explosion energy of the models.

\subsection{Different progenitors}

The analytic discussion of the previous section, in particular Eqs. (25), (27), and (28), allow us now to understand the behavior of the wind termination shock in different progenitor stars. For this purpose we compare our $15 M_{\odot}$ reference model, M1511-r1, with models M10-11-r1, M20-11-r1, and M25-15-r4, which are explosion simulations for $10.2,20$, and $25 M_{\odot}$ stars, respectively. The conditions at the inner grid boundary were chosen such that the models have similar explosion energies between roughly $1.3 \mathrm{~B}$ and $2 \mathrm{~B}$ (Table 2 ). The $25 M_{\odot}$ star has such a big mass accretion rate and correspondingly high accretion luminosity that the explosion tends to become stronger than in the lowermass progenitors. To lessen this effect, we reduced the boundary luminosities compared to the other models and chose a larger final radius of the inner boundary and thus of the new-born neutron star.

The neutron star mass and radius in the $10.2,15$, and $20 M_{\odot}$ simulations are rather similar (Table 2) and so are the timedependent luminosities, mean energies, and energy radiated in $v_{\mathrm{e}}$ and $\bar{v}_{\mathrm{e}}$, as well as the total energy release in neutrinos of all flavors, $\Delta E_{\text {tot }}$, (Fig. 7). The $25 M_{\odot}$ run, however, sticks out with significantly higher values of all these quantities. Progenitordependent differences associated with the density structure of the collapsing star outside of the iron core are responsible for the differences in the time-dependence of the explosion energy seen between the $10.2,15$, and $20 M_{\odot}$ models in the lower panel of Fig. 7. A more massive progenitor has a higher mass accretion rate and accretion luminosity and also a larger mass in the gain layer. Its explosion therefore tends to be more energetic. In case of the models M20-11-r1 and M25-15-r4, the large binding energy of the outer stellar shells later on leads to a visible decrease of the explosion energy from a maximum value reached transiently during the simulation (Fig. 7).

Figure 8 shows the time evolution of quantities that determine and characterize the neutrino wind and reverse shock behavior in our simulations with different progenitors. The wind properties (left column in Fig. 8) exhibit their well-known dependence on the neutron star mass and radius and on the neutrino luminosities and mean energies. Because of the similarity of these quantities in case of the $10.2,15$, and $20 M_{\odot}$ models, only rather small differences are visible between these runs, revealing a slightly longer expansion timescale, lower mass-loss rate, and higher entropy for model M20-11-r1 with its more massive neutron star (see also Table 2). The electron fraction shows a somewhat wider variation because of its strong sensitivity to the spectral and flux differences of the $v_{\mathrm{e}}$ and $\bar{v}_{\mathrm{e}}$ emission. The larger neutron star mass and neutrino luminosities in case of the $25 M_{\odot}$ progenitor separate this model clearly from the others. They affect in particular the neutrino-wind entropy, which scales with the value of the neutron star mass but is only weakly dependent on the neutrino emission properties (Eq. (16)). Nevertheless, since the neutron star is not extremely compact $\left(R_{\mathrm{ns}} \approx 11.5 \mathrm{~km}\right.$; Table 2$)$ and only moderately massive (gravitational mass $M_{\mathrm{grv}} \approx 1.66 M_{\odot}$ ), the wind entropy is never higher than $115 k_{\mathrm{B}}$ per nucleon during the 10 seconds of computed postbounce evolution. The expansion timescale and mass loss rate of the $25 M_{\odot}$ case are more similar to the other models because of a partial cancellation of their dependences on $L, \epsilon$, and $M$ in Eqs. (17) and (18).

The wind termination shock evolves largely differently in all cases (Fig. 8, right column). Obviously, the progenitor structure has a big influence on its behavior. The supernova shock expands much faster in the lower-mass stars, causing a more rapid decline of the pressure in the shell between forward and reverse shock. The propagation of the forward shock and the time-varying conditions there are communicated inward to the reverse shock on the sound propagation timescale. Therefore the pressure just downstream of the reverse shock, $P_{\mathrm{rs}}$, as well as the density and temperature at this location, decrease, too. From Eq. (28) it can be understood that in model M10-11-r1 the strong pressure reduction triggers a fast outward motion of the reverse shock. In the $15 M_{\odot}$ star the increase of $R_{\mathrm{rs}}$ is much less extreme, 

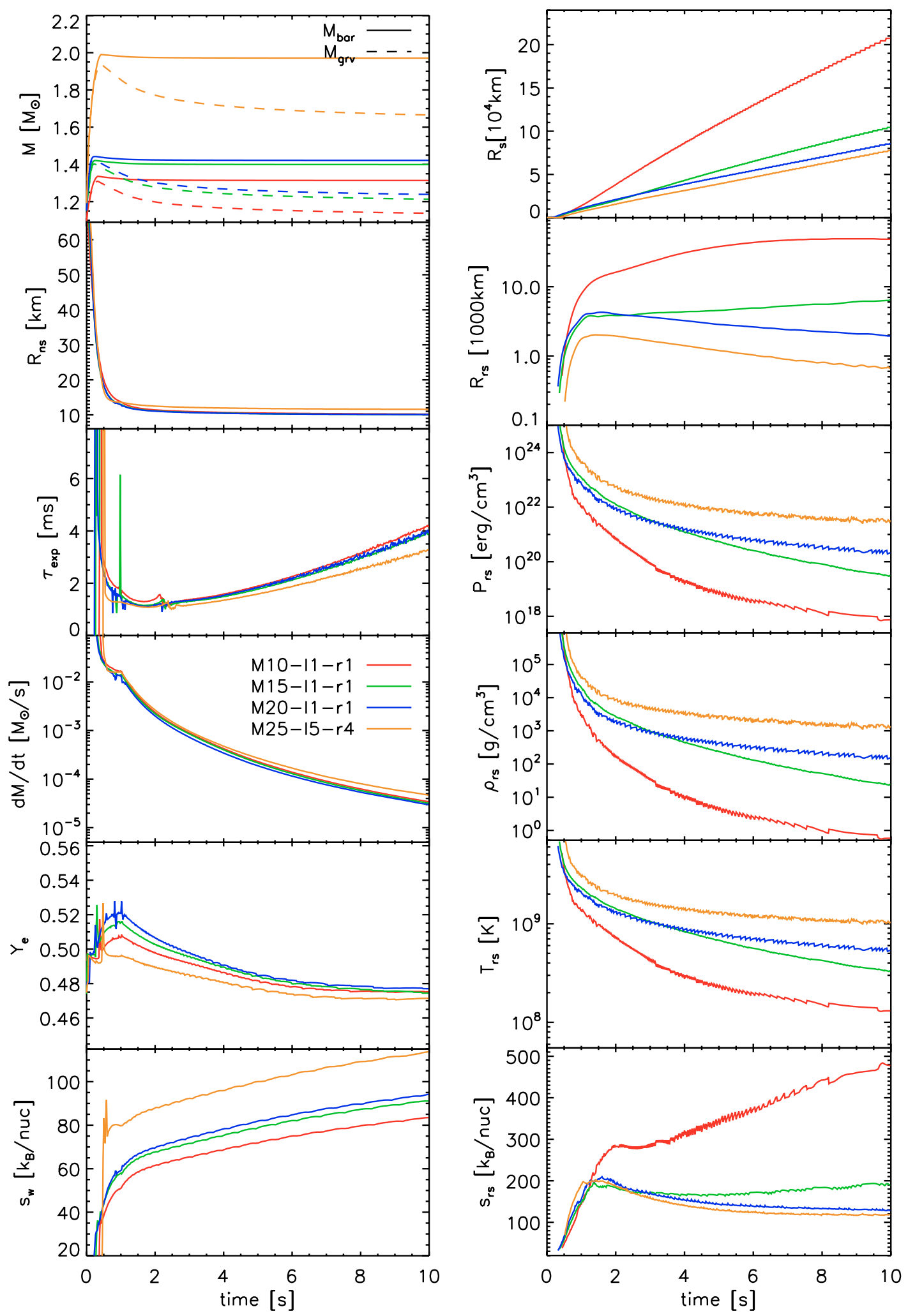

Fig. 8. Time evolution of different quantities for a set of simulations with different progenitor stars, M10-11-r1, M15-11-r1, M20-11-r1, and M2515-r4. Shown are the baryonic mass, $M_{\mathrm{bar}}$, and gravitational mass, $M_{\mathrm{grv}}$ (Eq. (7)), neutron star radius, neutrino-wind expansion timescale according to Eq. (15), wind mass-loss rate, electron fraction, and entropy per nucleon (left, from top to bottom), radius of the supernova shock, radius of the reverse shock, and pressure, density, temperature, and entropy per nucleon downstream of the reverse shock.

and in the 20 and $25 M_{\odot}$ runs the wind termination shock even retreats after $\sim 2 \mathrm{~s}$ of initial expansion and transient stagnation. In these cases the decline of $P_{\mathrm{rs}}$ is not fast enough to compete with the decrease of $\dot{M}_{\mathrm{w}}$ and $u_{\mathrm{w}}$ in the numerator of Eq. (28). A similar effect can be observed at $t=1 \mathrm{~s}$ when we change the time-dependence of the neutrino luminosity at the inner grid 


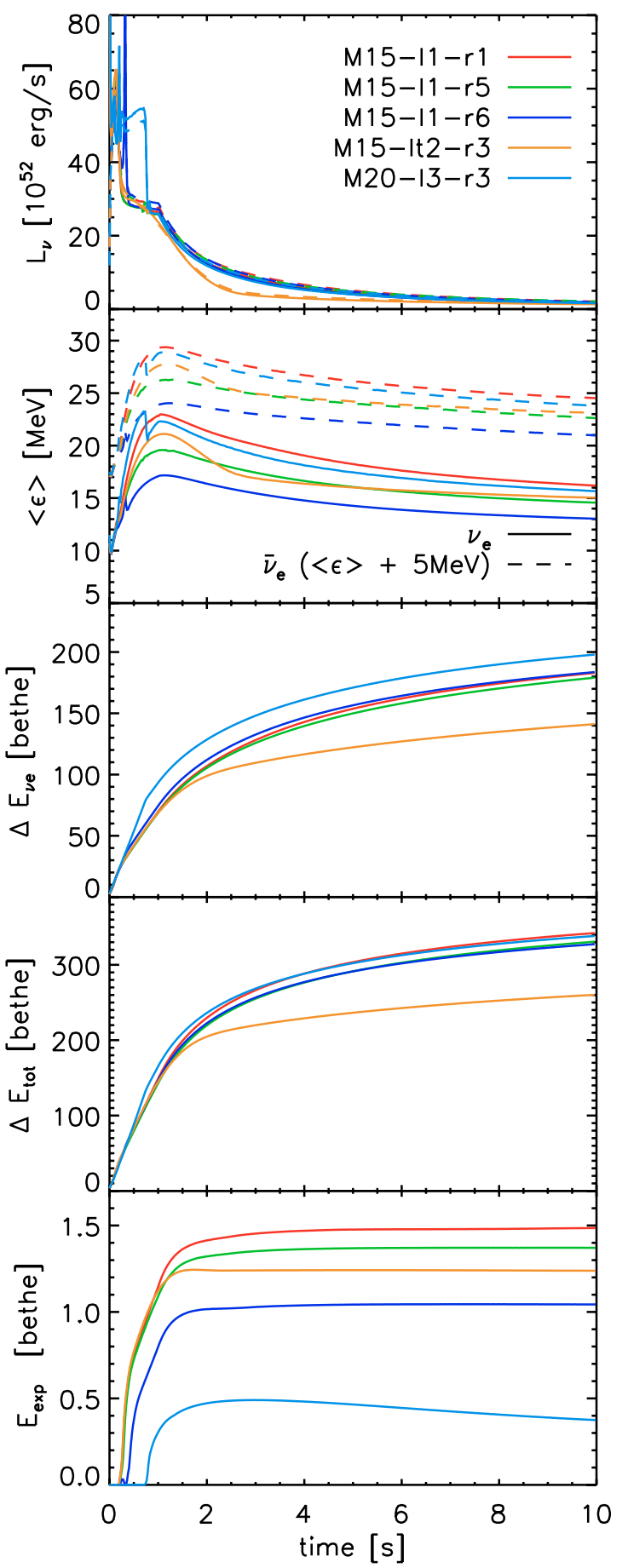

Fig. 9. The same as Fig. 7, but for simulations with varied inner boundary conditions (for clarity, however, we omitted the lines showing the rms energies of the radiated $v_{\mathrm{e}}$ and $\bar{v}_{\mathrm{e}}$ and shifted the $\bar{v}_{\mathrm{e}}$ energies by $5 \mathrm{MeV}$ ). The models M15-11-r5, M15-11-r6, M15-lt2-r3, and M20-13r3 are compared with our reference $15 M_{\odot}$ model M15-11-r1 in order to demonstrate the influence of different neutron star radii, neutron star masses, and core neutrino luminosities at the inner grid boundary.

boundary. The subsequent luminosity decrease leads to the mass-loss rate and velocity of the wind dropping more quickly than $P_{\mathrm{rs}}$ in all models except M10-11-r1, explaining why the initial expansion of the reverse shock is stopped at about this time.

Because of the different reverse shock behavior, the density, temperature, and entropy downstream of the reverse shock as functions of time show also large differences between the progenitors (Fig. 8). In model M10-11-r1 the wind termination shock moves to radii beyond $10000 \mathrm{~km}$ within little more than one second. During this phase the density $\rho_{\mathrm{w}}$ behind this shock drops to less than $10^{3} \mathrm{~g} \mathrm{~cm}^{-3}$ and the temperature $T_{\mathrm{w}}$ becomes lower than $10^{9} \mathrm{~K}$. The entropy, on the other hand, is nearly $300 k_{\mathrm{B}}$ per nucleon after 2 seconds. In the runs for the more massive progenitors, the density and temperature at the reverse shock are larger for a longer period of postbounce evolution, and the entropy does not reach the very high values of the $10 M_{\odot}$ simulation. The more massive the progenitor is - or, more precisely, the denser the shells around the iron core are - the slower propagates the shock for a given value of the explosion energy, and the more confined is the reverse shock. In none of the compared cases, however, are the conditions at the wind termination shock constant with time.

\subsection{Variations of inner boundary conditions}

It is clear from Eqs. (25), (27), and (28) that the behavior of the reverse shock does not only depend on the structure of the exploding star but also on the neutrino-wind properties, in particular the wind mass-loss rate and velocity. Since the latter increases with the distance from the neutron star, the radius of the reverse shock introduces an additional velocity dependence in Eqs. (25), (27), and (28).

In order to investigate the changes associated with different strength and time evolution of the neutrino wind, we varied the wind-determining parameters, i.e., the neutron star mass, radius, and contraction, and the core neutrino luminosities and energies as functions of time. In this section we therefore discuss the influence of these variations of the parameters used for the inner boundary condition.

The effect of the neutron star radius is visible from a comparison of our reference $15 M_{\odot}$ model M15-11-r1 with models M15-11-r5 and M15-11-r6 in Fig. 10. These three simulations are computed with the same inner boundary condition for the neutrinos and produce neutron stars with approximately the same gravitational masses but final radii of 10,13 , and roughly $17 \mathrm{~km}$, respectively (Table 2). The neutrino luminosities radiated from the nascent neutron star and the energy emitted in $v_{\mathrm{e}}$ and $\bar{v}_{\mathrm{e}}$ as well as the total energy lost in neutrinos are nearly the same (Fig. 9). Because of similar explosion energies, also the supernova shock in the three models propagates with similar velocity (Fig. 10).

The mean neutrino energies, however, show a clear correlation with the neutron star radius: the more compact the neutron star is, the higher are the energies of the escaping $v_{\mathrm{e}}$ and $\bar{v}_{\mathrm{e}}$ in Fig. 9. Also the neutrino-wind properties reveal the variation with the compactness of the neutron star that is qualitatively expected from the analytic expressions given by Qian \& Woosley (1996, see also Sect. 3.2, Eqs. (16)-(18)). A larger $R_{\mathrm{ns}}$ leads to a longer expansion timescale and thus lower wind velocity, larger mass-loss rate, and smaller wind entropy (see Fig. 10). In case of the mass-loss rate, however, the influence of the larger neutron star radius is partly cancelled by the lower mean neutrino energies (see Eq. (18)) (and by the slightly higher neutron star mass of model M15-11-r6), for which reason the differences in $\dot{M}$ are rather modest, in particular between models M15-11-r5 and M15-11-r6.

Qualitatively, the reverse shock exhibits the same behavior in these two models as in M15-11-r1. While its radius $R_{\mathrm{rs}}$ is essentially the same in models M15-11-r1 and M15-11-r5, the wind termination shock, however, expands less strongly in model M15-11-r6, reacting to the considerably lower wind 

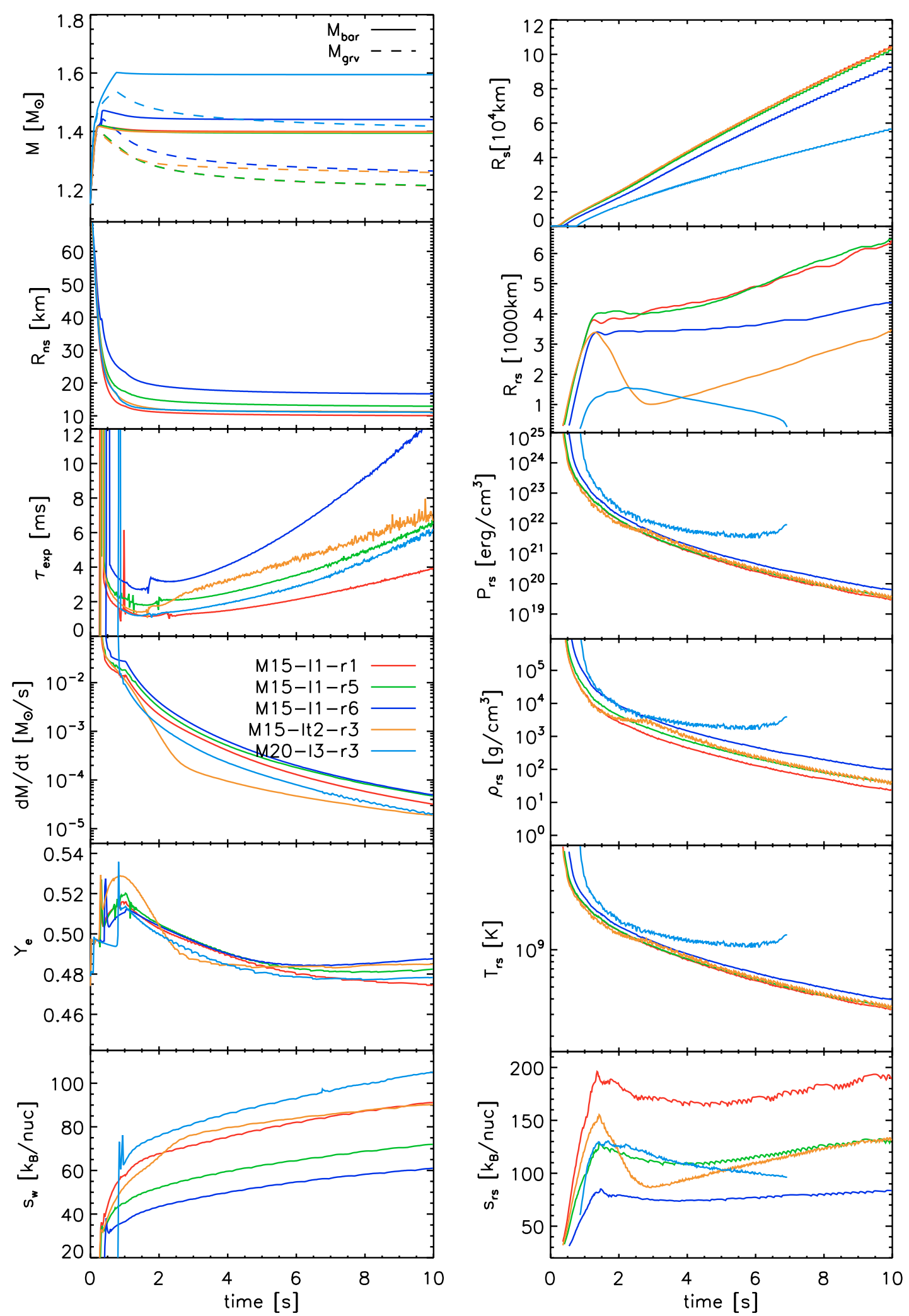

Fig. 10. The same as Fig. 8, but for simulations with varied inner boundary conditions. The models M15-11-r5, M15-11-r6, M15-lt2-r3, and M2013-r3 are compared with our reference $15 M_{\odot}$ model M15-11-r1 in order to demonstrate the influence of different neutron star radii, neutron star masses, and core neutrino luminosities at the inner grid boundary.

velocity and slightly slower propagation of the supernova shock in this somewhat less energetic model (cf. Eqs. (28) and (30)). Finally, the entropy of the matter decelerated in the reverse shock behaves as expected from Eqs. (25) and (27) when values for the wind parameters and reverse shock radius are inserted into these equations. It is highest in model M15-11-r1 and lowest in model M15-11-r6. The densities behind the reverse shock are ordered inversely. 
Model M15-lt2-r3 demonstrates the influence of a more rapid decay of the luminosities and mean energies of the radiated neutrinos after one second of postbounce evolution. This is associated with a reduced energy loss of the nascent neutron star and leads to an increase of the wind expansion timescale, a steep decrease of the wind mass loss rate, and a higher wind entropy compared to model M15-11-r1. The reverse shock reacts to that by a rapid recession between about $1 \mathrm{~s}$ and $3 \mathrm{~s}$ after bounce (cf. Eq. (28)) before it starts an outward motion again at later times when the pressure $P_{\text {rs }}$ drops faster than $\dot{M}_{\mathrm{w}}$ and $u_{\mathrm{w}}$ of the wind. Due to the small reverse shock radius, however, $u_{\mathrm{w}}$ at the shock is low and the entropy increase through the wind termination shock is modest.

Model M20-13-r3 with a neutron star radius and neutrino emission properties very similar to model M15-11-r1, but a significantly higher neutron star mass, reveals an even more extreme behavior. The larger neutron star mass increases the wind entropy, however at the same time reduces the wind mass-loss rate and the inverse wind expansion timescale (and thus the wind velocity; cf. Eqs. (16)-(18)). Moreover, the explosion energy of this model is very low and the supernova shock expands only slowly. All together forces the wind termination shock to retreat as the neutrino fluxes decay, until it falls below the sonic point in the wind and disappears. This brings the whole region from the proto-neutron star surface to the outer boundary of the neutrino-driven outflow (which is the contact discontinuity between shock-accelerated ejecta and neutrino-heated ejecta) in sonic contact, see Fig. 11. The neutrino-driven outflow is now only a subsonic breeze and merges with the dense shell of ejecta behind the outgoing supernova shock without being accelerated to supersonic speed.

In order to study this phenomenon and its implications in more detail, we triggered the occurrence of the breeze at a much earlier time in model M15-1t1-r4, in which the neutrino luminosities at the inner boundary were assumed to decay faster and the radiated neutrino energy is therefore even lower than in model M15-lt2-r3 (compare Fig. 12 with Fig. 9). As a consequence, the neutrino wind does not have sufficient power to keep the wind termination shock at a large radius. In spite of a standard explosion energy $\left(E_{\exp } \sim 1.2\right.$ bethe; Table 2 and Fig. 12) and fast propagation of the supernova shock, the reverse shock begins to retreat already after $1 \mathrm{~s}$ and disappears after $2.5 \mathrm{~s}$ (Fig. 11) whereas this happened only after $7 \mathrm{~s}$ as in model M15-lt2-r3 (Fig. 10). In Fig. 12 one sees that the transition to the subsonic breeze is accompanied by a considerable growth of the expansion timescales calculated from Eqs. (13) and (15). The timescale calculated from Eq. (14) exhibits even a sudden increase which occurs when the wind termination shock has retreated so much that it is encompassed by the radial integral of Eq. (14). The integral then includes shock-decelerated outflow which cools much more slowly.

After about $7 \mathrm{~s}$, however, the sound speed in the neutron star surroundings has dropped and the dense ejecta shell behind the supernova shock has moved outward sufficiently far that the neutrino-driven outflow can again reach supersonic velocities, despite much less powerful acceleration than in the first two seconds after bounce (Fig. 11). This is visible also in the radial profiles and mass shell trajectories plotted in Fig. 13, where at late times $(t \geq 8 \mathrm{~s})$ the discontinuity that characterizes the presence of a wind termination shock appears again in all quantities. Because of the meanwhile low wind velocity and very low massloss rate and therefore small reverse-shock radius, this shock is at late times much weaker than it was in the early phase. The associated density, temperature, and entropy steps are correspondingly small (Fig. 13).

During the breeze phase the outflow material is accelerated to a maximum velocity and then continuously decelerated again as it joins into the dense layer of ejecta behind the supernova shock. The mass-shell trajectories on the rhs. of Fig. 13 illustrate this smooth transition from the breeze expansion to the slower evolution when the matter is added to the dense ejecta shell.

Models M15-1t1-r4 and M20-13-r3 demonstrate clearly that the wind termination shock can be a transient feature and its presence is very sensitive to the time-dependent conditions in the neutrino-driven outflow and the expansion of the dense postshock shell of supernova ejecta. Simulations with a consistent treatment of the neutron star evolution and of the baryonic mass loss of the nascent neutron star are needed to make definitive predictions of the evolution of a given progenitor star. But even then such predictions are handicapped by our incomplete knowledge of the high-density equation of state and of the corresponding properties and neutrino emission of forming neutron stars.

\section{Possible implications for nucleosynthesis}

Our work had the goal to investigate the fundamental aspects of the neutrino-driven outflow from newly born neutron stars and of the wind termination shock that forms by the interaction of the supersonic wind with the shell of slower moving ejecta behind the supernova shock. We intended to explore the dependence of this reverse shock on the wind and the explosion properties in different progenitor stars. The outflow conditions in our models were found to differ significantly from the subsonic breeze solutions considered in many previous nucleosynthesis studies. Detailed network calculations are needed to analyse the consequences of these differences for the heavy-element formation in the outflows. Although this is beyond the scope of our present study, we want to add here a few speculative remarks about the possible implications.

\subsection{Shocked winds vs. unshocked winds and breezes}

Two main differences of the outflows in our models are potentially relevant for the assembling of heavy nuclei, in particular the formation of r-process elements in wind phases that develop a neutron excess. Firstly, the winds accelerate to supersonic speeds and therefore have shorter expansion timescales than breeze solutions, which by definition remain subsonic everywhere. This affects also the temperature range from $7 \times 10^{9} \mathrm{~K}$ down to $3 \times 10^{9} \mathrm{~K}$ that is crucial for the formation of seed nuclei from $\alpha$ particles and free nucleons through three-particle reactions (triple alpha and $\alpha \alpha \mathrm{n}$ ) and subsequent $\alpha$ captures. A faster expansion leads to less seed production and therefore an increase of the neutron-to-seed ratio. This increase might be significant for the modest values of the wind entropy obtained at the end of our simulations (we found up to $100-120 k_{\mathrm{B}}$ per nucleon, see Table 2, but $150 k_{\mathrm{B}}$ do not appear implausible at later times $t>10 \mathrm{~s}$ when the neutrino luminosities and mean energies have dropped further). Note that Takahashi et al. (1994), for example, employed breeze solutions with considerably longer expansion timescales in their nucleosynthesis studies.

Secondly, while the subsonic breezes gradually slow down after they have reached their maximum velocity, the wind matter going through the reverse shock is abruptly decelerated and its temperature, density, and entropy are increased. In the shocked flow the density and temperature then continue to drop on a 


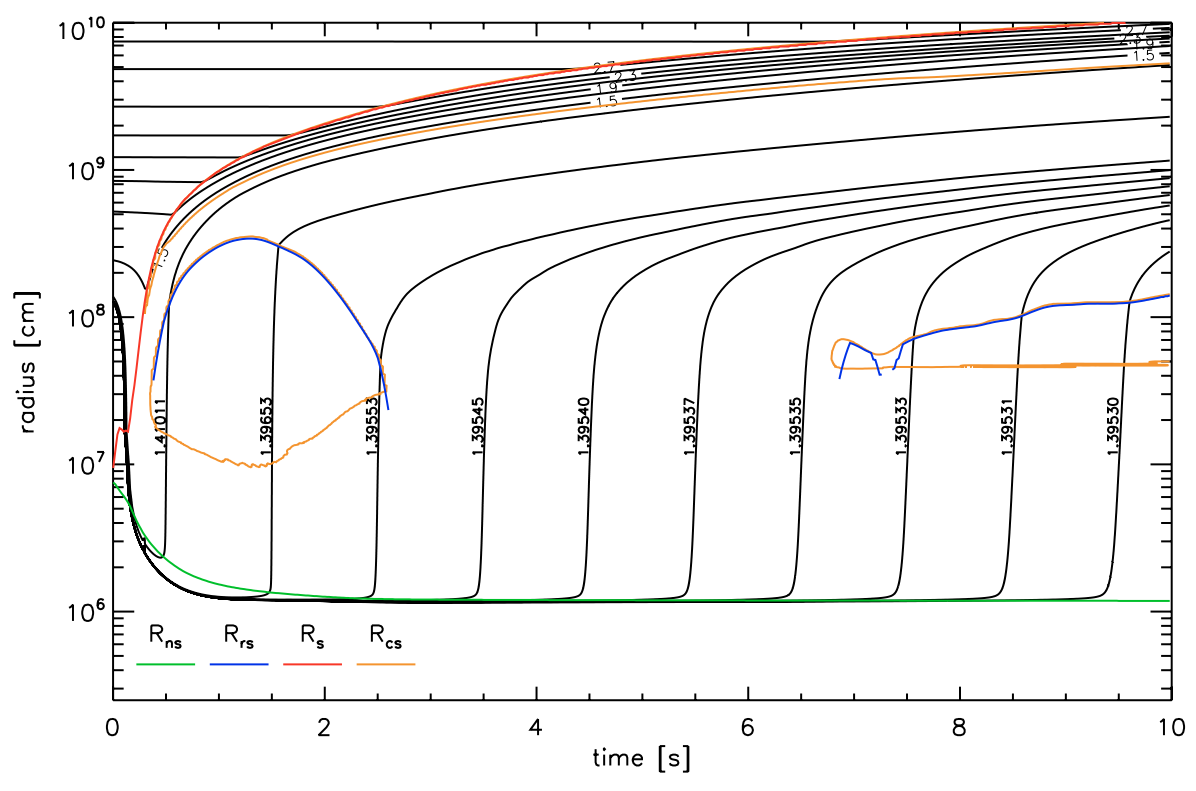

Fig. 11. Same as Fig. 3, but for model M15lt1-r4. Here the explosion occurs at $0.22 \mathrm{~s}$ after bounce and because of the assumed fast subsequent decay of the neutrino luminosity, the reverse shock reveals a much different behavior than in case of model M15-11-r1. It temporarily disappears when a subsonic breeze instead of a wind develops after about $2.5 \mathrm{~s}$. At $t \gtrsim 7 \mathrm{~s}$ the outflow expansion becomes supersonic again and a wind termination shock appears again.
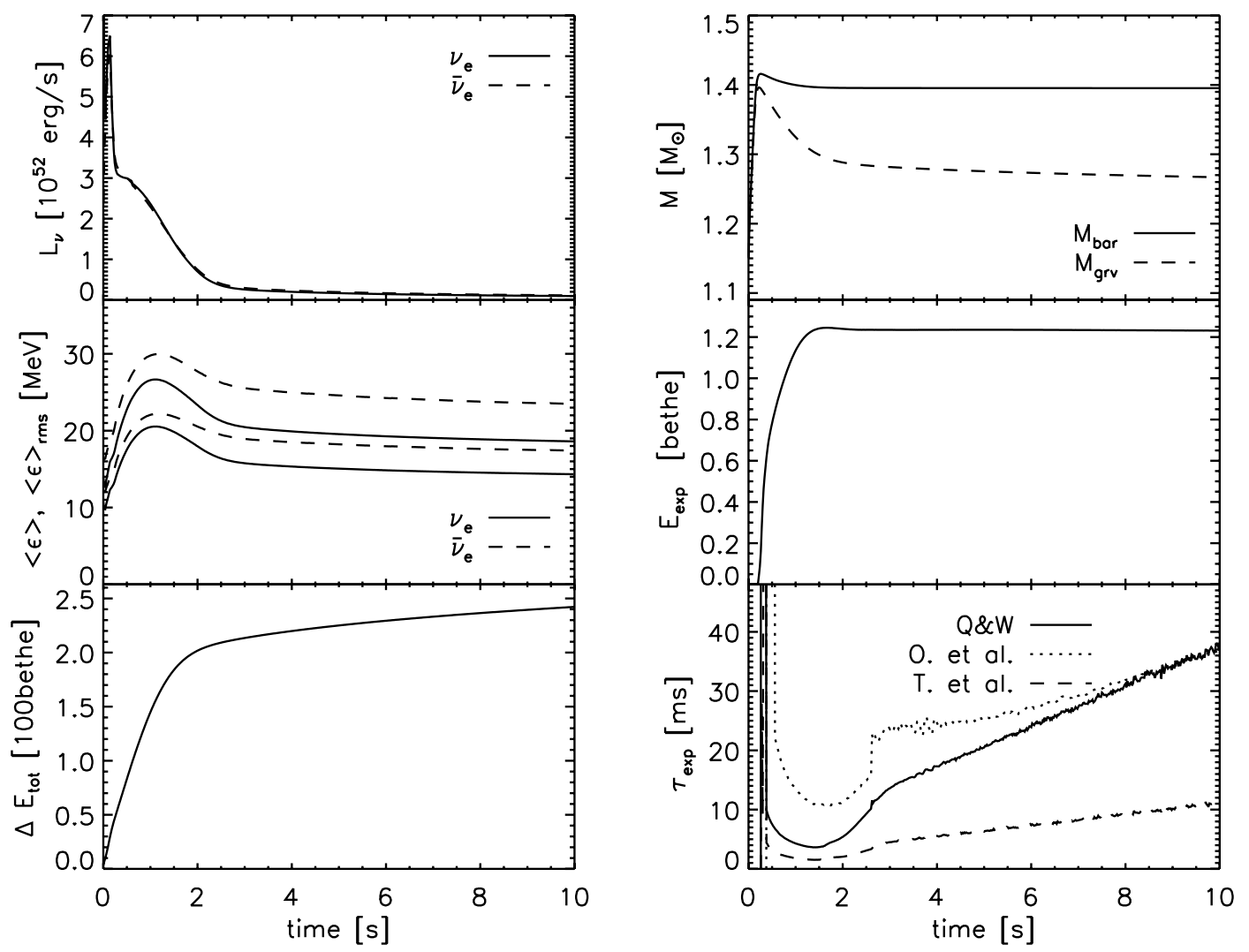

Fig. 12. Same as Fig. 4, but for model M15-1t1-r4. Compared to model M15-11-r1, the neutrino luminosities and mean energies decrease faster, the total energy radiated in neutrinos and the explosion energy are lower, and the gravitational mass of the neutron star is larger. The breeze solutions that develop between $2.5 \mathrm{~s}$ and $7 \mathrm{~s}$ have significantly longer expansion timescales.

much longer timescale than during the rapid early expansion of the wind. If the wind deceleration happens at conditions where free neutrons are still present - which is the case at temperatures around $10^{9} \mathrm{~K}$ in the breezes considered by Takahashi et al. (1994) but might be true at even lower temperatures in faster winds - the changed conditions may lead to a shift of the (n, $\gamma)$ $(\gamma, \mathrm{n})$ equilibrium and thus of the r-process path during the later stages of r-processing. This might alter the outcome of the nucleosynthesis compared to a freely expanding wind with its continuously growing velocity and compared to a gradually decelerated breeze. The results by Takahashi et al. (1994), see e.g. Fig. 2 there, suggest that the strength of the r-processing indeed depends on the dynamics of the outflow also at relatively low temperatures. In a most extreme situation (very rapid expansion, very high entropy) one might even imagine that neutrons remain unbound in the wind because their capture timescale becomes 

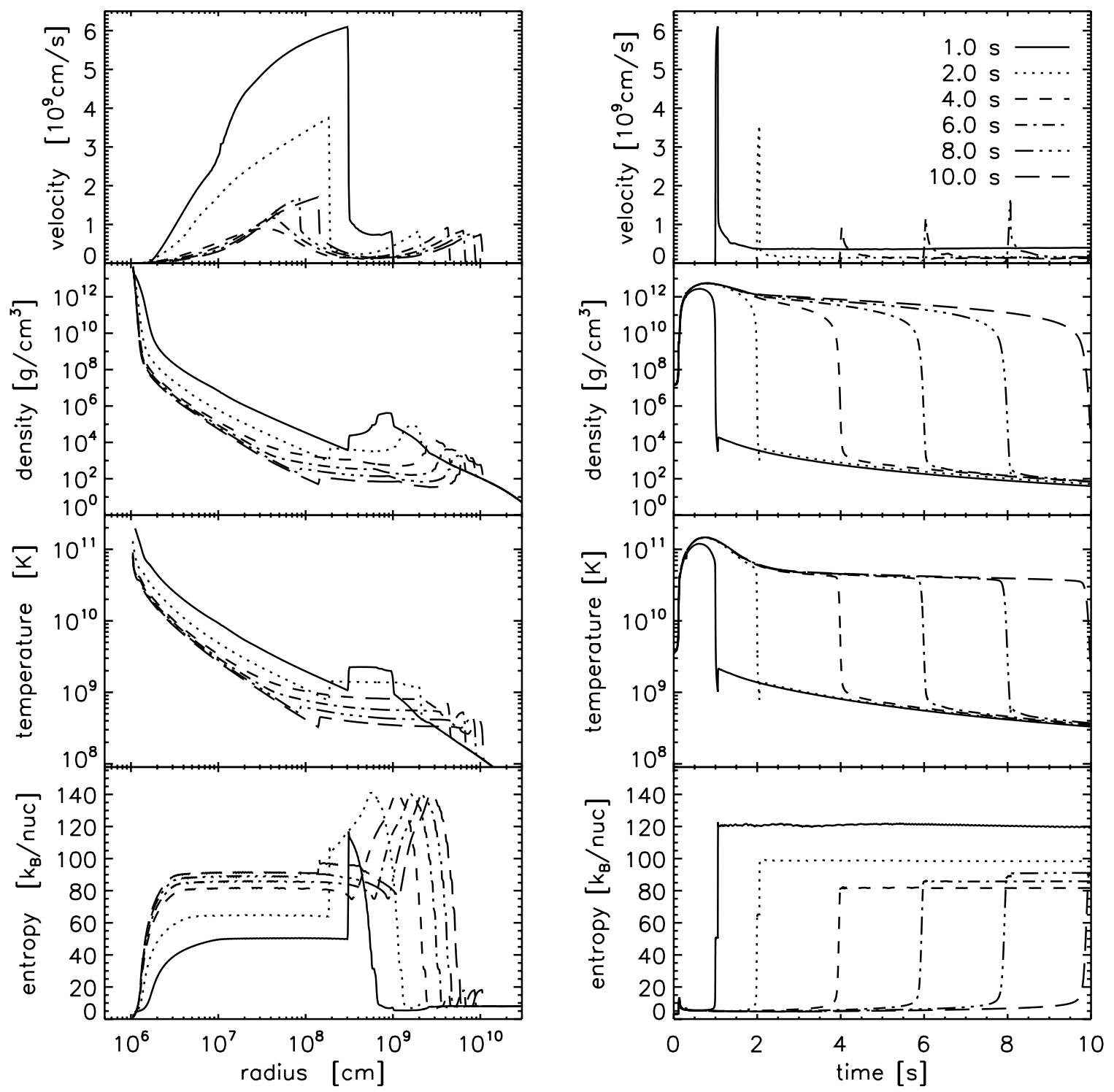

Fig. 13. Left, from top to bottom: radial profiles of the velocity, density, temperature, and entropy in model M15-lt1-r4 at different postbounce times. The profiles should be compared with the corresponding ones of model M15-11-r1 in Fig. 5. Right: the same quantities as functions of time for some mass shells ejected in the neutrino-driven outflow of model M15-lt1-r4. For comparison with Model M15-11-r1, see Fig. 6.

longer than the expansion timescale at some radius, whereas the shock-decelerated environment might give all neutrons enough time to be absorbed by seed nuclei.

Detailed network calculations are needed to clarify how strongly these differences really affect the heavy-element production in high-entropy supernova outflows for the conditions found in our models, and how the influence of these differences depends on the parameters that characterize the neutrino-driven wind and the reverse shock behavior. Such calculations being currently unavailable, we are not able to draw definitive conclusions here. In particular it is not clear whether indeed, and if so when, shock-decelerated supersonic winds can be more favorable for a strong r-process than previously studied breezes or unshocked winds. It is interesting, however, that Thompson et al. (2001) mentioned a strengthening of the production of thirdpeak r-process nuclei due to a slower postshock expansion and a shock-increased temperature at the time the r-process freeze-out happens.

\subsection{Progenitor trends}

We have presented simulations for a number of progenitor stars and have varied our prescription of the neutrino luminosities and of the neutron star radius and contraction with time. All of our choices for these conditions at the inner grid boundary were constrained by our need to obtain neutrino-driven explosions (and reasonable values for the explosion parameters) in spherically symmetric models. This required the assumption of sufficiently large boundary luminosities or could be achieved by assuming a more compact neutron star. The latter case leads to an enhanced release of gravitational binding energy and to increased neutrino fluxes and thus stronger neutrino heating behind the shock. The considered boundary settings were also motivated by the goal to demonstrate the different kinds of behavior that can occur for the wind termination shock, depending on possible variations of the properties of the neutrino-driven wind, of the explosion, and of the progenitor conditions. Identifying clearly the influence of individual aspects, however, is not an easy task in simulations 
like these, where the different components that play a role are strongly coupled.

Conclusions on systematic variations with the stellar progenitor are not only handicapped by the limited set of investigated models, but in particular also by the incomplete understanding of the supernova explosion mechanism and of the neutron star equation of state. This has the consequence that neither the explosion properties (energy, ejecta velocity, explosion timescale and thus mass cut) nor the properties of the compact remnant (e.g., its mass-radius relation and release of gravitational binding energy) can be reliably predicted at the present time, implying that the neutrino emission as a function of time (which may also be strongly influenced by convection inside the nascent neutron star) is not well known. This in turn means that not only the time evolution of the neutrino-driven wind is uncertain (cf. Eqs. (16)-(18)) but also the behavior and the influence of the wind termination shock is not definitely determined (due to the dependence of Eqs. (27) and (28) on the explosion and wind parameters). Additional complexity comes from the fact that multi-dimensional phenomena are important at least in some regions and during some phases of the evolution. Because of all these uncertainties, we concentrated here on a matter of principle study. Future simulations that include the neutrino cooling of the nascent neutron star instead of making use of our inner boundary condition will help to link and thus to reduce the involved degrees of freedom. Nevertheless, they will still have to test different nuclear equations of state and will have to make assumptions about the onset and the energetics of the supernova explosion.

In order to assess the resulting uncertainties in the prediction of the evolution of any particular progenitor model, we therefore investigated in Sect. 3.5 models where we varied the inner boundary conditions within a reasonable (but maybe somewhat "extreme") range of possibilities. In spite of these systematic uncertainties, we think that our "standard" (and preferred) set of models in Sect. 3.4 reveals some trends that either agree with expectations or are likely to be confirmed by more complete and more detailed future studies.

On the one hand, the neutron stars in more massive progenitors tend to be more massive, simply because of the larger stellar cores and the bigger mass infall rates after core bounce. This, of course, causes more energy release in neutrinos and higher neutrino fluxes for a longer period of time. Both the larger neutron star mass and the differences in the neutrino emission affect the neutrino-driven wind. In particular they lead to a tendency towards higher wind entropies for more massive progenitor stars (but, astonishingly, no significant differences in the wind expansion timescale and mass-loss rate as functions of time; see Fig. 8). On the other hand, more massive stars with their bigger and denser metal cores cause a less rapid expansion of the supernova shock during the computed phase of the explosion. The dense ejecta shell behind the supernova shock then forces the wind termination shock to follow this behavior (Fig. 8).

The consequence is that in less massive progenitors the reverse shock reaches large radii very quickly and continues to travel outward. Although it can lead to a huge increase of the entropy in the wind, because matter is decelerated by the termination shock from very large velocities, these effects happen after a short period of postbounce evolution only at a point where the temperature and density are already so low that the influence on the nucleosynthesis is probably marginal. In contrast, the reverse shock in more massive stars stays at a small radius during the whole $10 \mathrm{~s}$ of computed evolution and therefore for the most massive progenitors the reverse shock is endangered to retreat and even collapse at a later stage of the evolution when the neutrino-wind power has decreased. To see this happening, we would have to run our "standard" simulations for a significantly longer evolution period than just 10 seconds (with substantial demands on computer time because of time-step limitations and the need to continuously increase the numerical resolution). The fundamental possibility of a contraction of the reverse shock, however, could be demonstrated by varying the boundary conditions in some of our models such that the neutrino luminosities and neutrino-wind power had dropped sufficiently already within the canonical $10 \mathrm{~s}$ of computed evolution (Sect. 3.5). The reverse shock staying at a small radius causes a more moderate increase of the wind entropy but still decelerates the supersonic wind abruptly and raises the density and temperature of the expanding matter. These discontinuous changes of the outflow conditions are time-dependent and might have interesting but so far unexplored consequences for the nucleosynthesis.

We therefore conclude that the neutrino-driven outflows in our models are supersonic winds in which the nucleosynthesisrelevant conditions are similar to the wind solutions investigated previously (e.g., by Thompson et al. 2001). However, these winds are bounded at some radius by the termination shock, which modifies the outflow behavior drastically. In lowmass supernova progenitors this reverse shock is likely to have nucleosynthesis-relevant consequences only during the first few seconds after bounce. For more massive progenitors the winds are abruptly decelerated, compressed, and heated by the wind termination shock during a stage of their expansion (i.e. at a density and temperature) where the r-process path might be affected. The combination of very rapidly expanding and ultimately supersonic wind and a termination shock that changes the evolution of the outflow, has so far not been investigated in detail for its nucleosynthesis implications. In progenitors with very massive and dense metal cores (roughly for stars with $\gtrsim 25 M_{\odot}$ ), which can give birth to neutron stars with a mass of $\gtrsim 2 M_{\odot}$, the wind termination shock might recede and possibly disappear so that the outflow develops into a subsonic breeze instead of being a supersonic wind. In this case the nucleosynthesis environment becomes more similar to the conditions studied by Takahashi et al. (1994), Sumiyoshi et al. (2000) or Otsuki et al. (2000) where subsonic ejecta reach a maximum velocity and then slow down gradually to meet an imposed boundary condition at a certain radius. This boundary condition is supposed to account for the presence of the dense shell of ejecta that follows the expanding supernova shock and that absorbs the later neutrino-driven outflow from the cooling neutron star. Our models show that in a supernova core this "boundary" is time dependent and its consequences are not likely to be well described by the simple (e.g. constant) assumptions made for the idealized neutrino-driven outflows considered in many nucleosynthesis studies.

Our simulations therefore suggest that an analysis of the nucleosynthesis based on more detailed models of the conditions in supernova cores is very desirable. If the reverse shock feature has a direct relevance for the possibility of r-processing, its biggest impact must be expected for progenitors between roughly $15 M_{\odot}$ and roughly $25 M_{\odot}$, where the wind termination shock is neither at too large radii nor disappears during the evolution phases that are most favorable for a strong r-process.

\section{Summary and conclusions}

We have presented long-time 1D hydrodynamic simulations of neutrino-driven explosions and post-explosion outflows for progenitors with different masses. In these simulations the core 
of the shrinking nascent neutron star at neutrino optical depths larger than about 100 was replaced by an inner boundary whose contraction was prescribed and where time-dependent neutrino fluxes were imposed such that the neutrino-energy deposition around the neutron star produced explosions with a desired energy. The time-dependence of both the boundary motion and of the neutrino luminosities was varied to investigate their influence on the high-entropy baryonic outflow and its interaction with the preceding supernova ejecta. Solving the Newtonian equations of hydrodynamics, we included approximately the effects of relativistic gravity by employing the "effective relativistic potential" of Marek et al. (2006). This approximation yields very good agreement with fully relativistic calculations during the postbounce accretion phase, and we found also nice consistency of our approach with fully relativistic solutions of stationary neutrino winds.

For the neutrino transport we performed a radial integration of the frequency-integrated energy and lepton number equations, in which the neutrino spectra were assumed to have a FermiDirac non-equilibrium shape in the sense that the neutrino spectral temperature could differ from the temperature of the stellar medium (see Scheck et al. 2006). The neutrino luminosities and mean energies are thus functions of time as well as radius in our simulations.

Because of the involved approximations and assumptions, our calculations can only be suggestive but are not suitable for definitive predictions of the nucleosynthesis-relevant conditions in dependence of the progenitor star. Our main goal was therefore a matter-of-principle study of the interaction of the neutrinodriven baryonic outflow from the neutron star surface with the slower dense shell of ejecta that is accelerated by the outgoing supernova shock. The most important results are the following:

- All of our models develop supersonic winds at least during some phases of their evolution. These winds are bounded by a termination shock in which the flow is abruptly decelerated and the entropy, density, and temperature of the flow are strongly increased.

- The basic properties of this wind termination shock can be understood from simple analytic considerations using the shock-jump conditions at this reverse shock. The entropy of the shock-decelerated matter increases with the wind velocity and is lower for high wind density. Therefore a large reverse shock radius is favorable for a high entropy jump. The reverse shock radius increases with the mass-loss rate and velocity of the wind, but decreases when the pressure behind the reverse shock is high. The latter dependence links the behavior of the reverse shock to the propagation of the supernova shock and thus to the progenitor structure and the explosion properties.

- The conditions at the reverse shock are progenitor-dependent and usually strongly time-dependent and therefore the shock effects are not well represented by an outer boundary condition with constant pressure (e.g., Sumiyoshi et al. 2000) or constant temperature (e.g., Wanajo et al. 2002). The conversion of kinetic energy to internal energy in the wind termination shock can raise the entropy to several times the wind entropy. We found the highest values (until $10 \mathrm{~s}$ of postbounce evolution) of nearly $500 k_{\mathrm{B}}$ per nucleon behind the reverse shock (more than a factor of five increase) - but also the lowest temperatures $\left(\$ 10^{9} \mathrm{~K}\right)$ and densities $\left(\$ 1000 \mathrm{~g} \mathrm{~cm}^{-3}\right)-$ in case of the considered $10 M_{\odot}$ progenitor. In this star the supernova shock and the reverse shock propagate outward very rapidly. In the considered progenitors with masses of more than $15 M_{\odot}$ the maximum entropies are higher than $200 k_{\mathrm{B}}$ per nucleon, corresponding to an increase of roughly a factor of three, with densities and temperatures behind the reverse shock in the first ten seconds of typically $100-10^{4} \mathrm{~g} \mathrm{~cm}^{-3}$ and $0.4-2 \times 10^{9} \mathrm{~K}$, respectively.

- When the supernova shock expands slowly (as in the case of very massive progenitors with big and dense metal cores) or the neutrino emission from the nascent neutron star decays rapidly and the wind power thus drops quickly, the reverse shock can show phases of recession and can even fall below the sonic point in the wind. The outflow then becomes a subsonic breeze that merges smoothly with the ejecta shell behind the shock without any jumps in the velocity and in the thermodynamic quantities. Changing conditions around the neutron star can lead to a re-establishment of a supersonic wind at later times.

The consequences of the deceleration of the neutrino-driven wind or breeze by the dense shell behind the supernova shock were parametrized previously in many r-process studies by imposing a mostly time-independent outer boundary condition in hydrodynamic models (e.g., Sumiyoshi et al. 2000, Terasawa et al. 2002) or by selecting those solutions of the steady-state equations that fulfill certain conditions (e.g., Otsuki et al. 2000; Wanajo et al. 2001, 2002). Here, however, we have demonstrated that such prescriptions do in general not adequately account for the effects that happen when the neutrino-driven outflow collides with the preceding, slower postshock shell. A wind termination shock can not only alter the wind entropy, density, and temperature by factors of a few, but also leads to a much slower expansion of the shocked outflow after its deceleration. It will have to be explored in detail by nucleosynthesis calculations how the combination of the very rapid expansion of the supersonic winds and the changing conditions in the matter due to the reverse shock affect heavy-element formation in the high-entropy supernova ejecta.

All of our simulations exhibit the presence of proton-rich conditions in the neutrino-driven outflow during the first $2-3 \mathrm{~s}$ of the explosion. Only later a neutron excess develops in the wind matter. Our result of $Y_{\mathrm{e}}>0.5$ in the early neutrino-driven wind is qualitatively in agreement with models that employ Boltzmann neutrino transport (Buras et al. 2006b; Pruet et al. 2005; Fröhlich et al. 2006). We point out, however, that quantitatively meaningful calculations of the proton-to-neutron ratio require frequencydependent neutrino transport. Moreover, a reliable prediction of the turning point from proton excess to neutron excess and of the value of $Y_{\mathrm{e}}$ at late postbounce times is not possible without fully consistent cooling calculations of the proto-neutron star instead of our inner grid boundary with prescribed time dependence of the neutrino fluxes. At best, our calculations can be indicative for the trends which will also be found in such improved 1D models.

The varying conditions in the neutrino-driven wind and the strong time- and progenitor-dependence of the behavior of the wind termination shock and of its effects on the wind raise a serious question: Do supernova cores provide the robust environment for producing the extremely uniform solar-system like r-process abundance pattern between the Ba- and Pt-peaks observed in ultra metal-poor stars (see, e.g., Cowan \& Sneden 2006)? In addition to the variations that are present in our models, multi-dimensional effects lead to long-lasting anisotropic accretion and at the same time directed outflows (e.g., Scheck et al. 2006). These introduce a stochastic element in the supernova evolution during the first seconds of postbounce evolution (see also Burrows et al. 2006, 2007). The supernova ejecta in 
different directions can develop largely different conditions due to the strong anisotropy of the explosion mechanism and of the environment of the forming neutron star. Downdrafts and pockets of dense, low-entropy matter in the convective shell behind the forward shock, for example, lag behind the overall outward expansion and cause a large-scale deformation of the reverse shock that terminates the supersonic neutrino-driven wind also in the multi-dimensional case. The varying radius and orientation of the reverse shock relative to the radial direction lead to an angular dependence of the properties of the shocked matter. Therefore the amount of matter ejected with certain conditions (e.g., entropy, expansion timescale after passing the shock) differs between spherically symmetric and multi-dimensional models. A detailed hydrodynamic study in $2 \mathrm{D}$ is currently underway (Arcones et al. 2007).

It is hard to see how this chaotic variability can allow for the robustness of environmental conditions needed for producing a uniform abundance pattern of high-mass r-process elements (even if some chunks of possible ejecta achieve to develop suitable conditions of high entropy and low $Y_{\mathrm{e}}$ as observed in the recent models of Burrows et al. 2007). If supernovae are the main sources of the high-mass nuclei beyond the $A \sim 130$ abundance peak - and a number of arguments have been made in support of that (e.g., Cowan \& Thielemann 2004) - a solution of this puzzle may be that these nuclei are produced in the later stages of the neutrino-driven wind, which are unaffected by the turbulent initial phase of the explosion. The proton-richness of the early supernova ejecta seen in our models in agreement with stateof-the-art simulations with energy-dependent neutrino transport, and the transition to n-richness at later times, yield support for this argument. The spherically symmetric supersonic winds with their wind termination shocks simulated in this work may be more characteristic of these late stages than of the early, still turbulent phases of the explosion. Previous investigations, however, suggest that the conditions in (unshocked) neutrino-driven outflows are insufficient for a strong r-processing unless the neutron star is very massive (around $\left.2 M_{\odot}\right)$ and very compact $(\sim 9 \mathrm{~km}$; e.g., Thompson et al. 2001; Otsuki et al. 2000).

It is currently not clear whether the supersonic outflows in our more "realistic" explosion models with the presence of the reverse shock can ease this constraint and help establishing an r-process favorable environment for less extreme assumptions of neutron star mass and radius. Maybe this is realised only in a subset of progenitors where the reverse shock is at a beneficial location. Such a requirement could single out rather discontinuously progenitors with particular core and explosion properties as favorable, while other progenitors do not develop suitable conditions for a strong r-processing. More simulations, improved modeling, and detailed nucleosynthesis calculations are needed to explore such possibilities.

Acknowledgements. We are grateful to S. Woosley and A. Heger for providing us with their progenitor models, to A. Marek for computing collapse and prompt shock propagation phases with the VERTEX neutrino-hydrodynamics code, and to R. Buras for assisting A.A. in implementing and testing the effective relativistic potential in the code with inner grid boundary. We also thank K. Langanke, G. Martínez-Pinedo, and I. Panov for stimulating discussions. Support by the Sonderforschungsbereich 375 on "Astro-Particle Physics" of the Deutsche Forschungsgemeinschaft is acknowledged. The computations were performed on the NEC SX-5/3C and the IBM p690 "Regatta" of the
Rechenzentrum Garching, and on the IBM p690 "Jump" of the John von Neumann Institute for Computing in Jülich.

\section{References}

Arcones, A., Janka, H.-T., \& Scheck, L. 2007, in preparation

Buras, R., Rampp, M., Janka, H.-T., \& Kifonidis, K. 2003, Phys. Rev. Lett. 90, 241101

Buras, R., Janka, H.-T., Rampp, M., \& Kifonidis, K. 2006a, A\&A, 457, 281

Buras, R., Rampp, M., Janka, H.-T., \& Kifonidis, K. 2006b, A\&A, 447, 1049

Burrows, A., \& Lattimer, J. M. 1986, ApJ, 307, 178

Burrows, A., Hayes, J., \& Fryxell, B. A. 1995, ApJ, 450, 830

Burrows, A., Livne, E., Dessart, L., Ott, C. D., \& Murphy, J. 2006, ApJ, 640, 878

Burrows, A., Livne, E., Dessart, L., Ott, C. D., \& Murphy, J. 2007, ApJ, 655, 416

Cardall, C. Y., \& Fuller, G. M. 1997, ApJ, 486, L111

Colella, P., \& Woodward, P. R. 1984, J. Comp. Phys. 54, 174

Cowan, J. J., \& Sneden, C. 2006, Nature, 440, 1151

Cowan, J. J., \& Thielemann, F.-K. 2004, Physics Today, 57, 47

Duncan, R. C., Shapiro, S. L., \& Wasserman, I. 1986, ApJ, 309, 141

Fröhlich, C., et al. 2006, ApJ, 637, 415

Heger, A., Woosley, S. E., Martínez-Pinedo, G., \& Langanke, K. 2001, ApJ, 560, 307

Hoffman, R. D., Woosley, S. E., Fuller, G. M., \& Meyer, B. S. 1996, ApJ, 460, 478

Janka, H.-T. 1991, A\&A, 244, 378

Janka, H.-T., \& Müller, E. 1995, ApJ, 448, L109

Janka, H.-T., \& Müller, E. 1996, A\&A, 306, 167

Keil, W., \& Janka, H.-T. 1995, A\&A, 296, 145

Kifonidis, K., Plewa, T., Janka, H.-T., \& Müller, E. 2003, A\&A, 408, 621

Lattimer, J. M., \& Swesty, F. 1991, Nucl. Phys. A, 535, 331

Liebendörfer, M., Rampp, M., Janka, H.-T., \& Mezzacappa, A. 2005, ApJ, 620, 840

Marek, A., Dimmelmeier, H., Janka, H.-T., Müller, E., \& Buras, R. 2006, A\&A, 445,273

Metzger, B. D., Thompson, T. A., \& Quataert, E. 2006, ApJ, submitted [arXiv:astro-ph/0608682]

Meyer, B. S. 1994, ARA\&A, 32, 153

Meyer, B. S., Mathews, G. J., Howard, W. M., Woosley, S. E., \& Hoffman, R. D. 1992, ApJ, 399, 656

Otsuki, K., Tagoshi, H., Kajino, T., \& Wanajo, S. 2000, ApJ, 533, 424

Pons, J. A., Reddy, S., Prakash, M., Lattimer, J. M., \& Miralles, J. A. 1999, ApJ, 513, 780

Pruet, J., Woosley, S. E., Buras, R., Janka, H.-T., \& Hoffman, R. D. 2005, ApJ, 623,325

Qian, Y.-Z., \& Woosley, S. E. 1996, ApJ, 471, 331

Rampp, M., \& Janka, H.-T. 2002, A\&A, 396, 361

Scheck, L., Plewa, T., Janka, H.-T., Kifonidis, K., \& Müller, E. 2004, Phys. Rev. Lett., 92, 011103

Scheck, L., Kifonidis, K., Janka, H.-T., \& Müller, E. 2006, A\&A, 457, 963

Sumiyoshi, K., Suzuki, H., Otsuki, K., Terasawa, M., \& Yamada, S. 2000, PASJ, 52,601

Takahashi, K., \& Janka, H.-T. 1997, in Origin of matter and evolution of galaxies in the universe '96, ed. T. Kajino, Y. Yoshii, \& S. Kubono, 213

Takahashi, K., Witti, J., \& Janka, H.-T. 1994, A\&A, 286, 857

Terasawa, M., Sumiyoshi, K., Yamada, S., Suzuki, H., \& Kajino, T. 2002, ApJ, 578, L137

Thompson, T. A., Burrows, A., \& Meyer, B. S. 2001, ApJ, 562, 887

Wanajo, S. 2006, ApJ, 650, L79

Wanajo, S., Itoh, N., Ishimaru, Y., Nozawa, S., \& Beers, T. C. 2002, ApJ, 577, 853

Wanajo, S., Kajino, T., Mathews, G. J., \& Otsuki, K. 2001, ApJ, 554, 578

Witti, J., Janka, H.-T., \& Takahashi, K. 1994, A\&A, 286, 841

Woosley, S. E., \& Baron, E. 1992, ApJ, 391, 228

Woosley, S. E., Heger, A., \& Weaver, T. A. 2002, Rev. Mod. Phys., 74, 1015

Woosley, S. E., \& Hoffman, R. D. 1992, ApJ, 395, 202

Woosley, S. E., \& Weaver, T. A. 1995, ApJS, 101, 181

Woosley, S. E., Wilson, J. R., Mathews, G. J., Hoffman, R. D., \& Meyer, B. S. 1994, ApJ, 433, 229 